\title{
Combination Strategies for Semantic Role Labeling
}

\author{
Mihai Surdeanu \\ Lluís Màrquez \\ Xavier Carreras \\ Pere R. Comas \\ Technical University of Catalonia, \\ C/ Jordi Girona, 1-3 \\ 08034 Barcelona, SPAIN
}

\author{
SURDEANU@LSI.UPC.EDU \\ LLUISM@LSI.UPC.EDU \\ CARRERAS@LSI.UPC.EDU \\ PCOMAS@LSI.UPC.EDU
}

\begin{abstract}
This paper introduces and analyzes a battery of inference models for the problem of semantic role labeling: one based on constraint satisfaction, and several strategies that model the inference as a meta-learning problem using discriminative classifiers. These classifiers are developed with a rich set of novel features that encode proposition and sentence-level information. To our knowledge, this is the first work that: (a) performs a thorough analysis of learning-based inference models for semantic role labeling, and (b) compares several inference strategies in this context. We evaluate the proposed inference strategies in the framework of the CoNLL-2005 shared task using only automatically-generated syntactic information. The extensive experimental evaluation and analysis indicates that all the proposed inference strategies are successful - they all outperform the current best results reported in the CoNLL-2005 evaluation exercise- but each of the proposed approaches has its advantages and disadvantages. Several important traits of a state-of-the-art SRL combination strategy emerge from this analysis: (i) individual models should be combined at the granularity of candidate arguments rather than at the granularity of complete solutions; (ii) the best combination strategy uses an inference model based in learning; and (iii) the learning-based inference benefits from max-margin classifiers and global feedback.
\end{abstract}

\section{Introduction}

Natural Language Understanding (NLU) is a subfield of Artificial Intelligence (AI) that deals with the extraction of the semantic information available in natural language texts. This knowledge is used to develop high-level applications requiring textual and document understanding, such as Question Answering or Information Extraction. NLU is a complex "AI-complete" problem that needs to venture well beyond the syntactic analysis of natural language texts. While the state of the art in NLU is still far from reaching its goals, recent research has made important progress in a subtask of NLU: Semantic Role Labeling. The task of Semantic Role Labeling (SRL) is the process of detecting basic event structures such as who did what to whom, when and where. See Figure 1 for a sample sentence annotated with such an event frame.

\subsection{Motivation}

SRL has received considerable interest in the past few years (Gildea \& Jurafsky, 2002; Surdeanu, Harabagiu, Williams, \& Aarseth, 2003; Xue \& Palmer, 2004; Pradhan, Ha- 
cioglu, Krugler, Ward, Martin, \& Jurafsky, 2005a; Carreras \& Màrquez, 2005). It was shown that the identification of such event frames has a significant contribution for many NLU applications such as Information Extraction (Surdeanu et al., 2003), Question Answering (Narayanan \& Harabagiu, 2004), Machine Translation (Boas, 2002), Summarization (Melli, Wang, Liu, Kashani, Shi, Gu, Sarkar, \& Popowich, 2005), and Coreference Resolution (Ponzetto \& Strube, 2006b, 2006a).

From a syntactic perspective, most machine-learning SRL approaches can be classified in one of two classes: approaches that take advantage of complete syntactic analysis of text, pioneered by Gildea and Jurafsky (2002), and approaches that use partial syntactic analysis, championed by previous evaluations performed within the Conference on Computational Natural Language Learning (CoNLL) (Carreras \& Màrquez, 2004, 2005). The wisdom extracted from the first representation indicates that full syntactic analysis has a significant contribution to SRL performance, when using hand-corrected syntactic information (Gildea \& Palmer, 2002). On the other hand, when only automatically-generated syntax is available, the quality of the information provided through full syntax decreases because the state-ofthe-art of full parsing is less robust and performs worse than the tools used for partial syntactic analysis. Under such real-world conditions, the difference between the two SRL approaches (with full or partial syntax) is not that high. More interestingly, the two SRL strategies perform better for different semantic roles. For example, models that use full syntax recognize agent and theme roles better, whereas models based on partial syntax are better at recognizing explicit patient roles, which tend to be farther from the predicate and accumulate more parsing errors (Màrquez, Comas, Giménez, \& Català, 2005).

\subsection{Approach}

In this article we explore the implications of the above observations by studying strategies for combining the output of several independent SRL systems, which take advantage of different syntactic views of the text. In a given sentence, our combination models receive labeled arguments from individual systems, and produce an overall argument structure for the corresponding sentence. The proposed combination strategies exploit several levels of information: local and global features (from individual models) and constraints on the argument structure. In this work, we investigate three different approaches:

- The first combination model has no parameters to estimate; it only makes use of the argument probabilities output by the individual models and constraints over argument structures to build the overall solution for each sentence. We call this model inference with constraint satisfaction.

- The second approach implements a cascaded inference model with local learning: first, for each type of argument, a classifier trained offline decides whether a candidate is or is not a final argument. Next, the candidates that passed the previous step are combined into a solution consistent with the constraints over argument structures. We refer to this model as inference with local learning.

- The third inference model is global: a number of online ranking functions, one for each argument type, are trained to score argument candidates so that the correct argument structure for the complete sentence is globally ranked at the top. We call this model inference with global learning. 


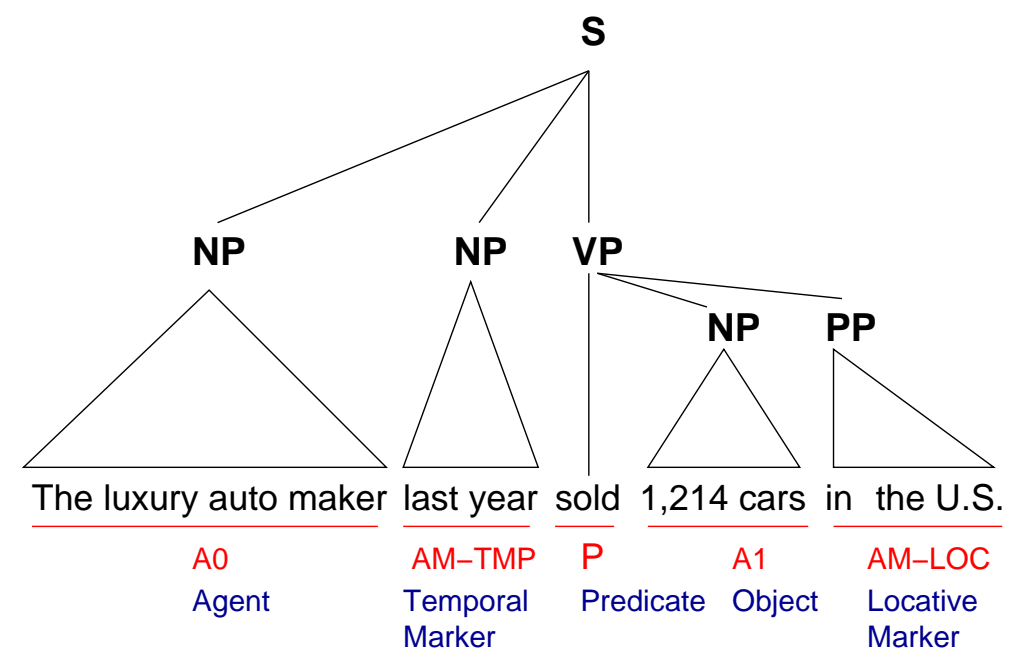

Figure 1: Sample sentence from the PropBank corpus.

The proposed combination strategies are general and do not depend on the way in which candidate arguments are collected. We empirically prove it by experimenting not only with individual SRL systems developed in house, but also with the 10 best systems at the CoNLL-2005 shared task evaluation.

\subsection{Contribution}

The work introduced in this paper has several novel points. To our knowledge, this is the first work that thoroughly explores an inference model based on meta-learning (the second and third inference models introduced) in the context of SRL. We investigate metalearning combination strategies based on rich, global representations in the form of local and global features, and in the form of structural constraints of solutions. Our empirical analysis indicates that these combination strategies outperform the current state of the art. Note that all the combination strategies proposed in this paper are not "re-ranking" approaches (Haghighi, Toutanova, \& Manning, 2005; Collins, 2000). Whereas re-ranking selects the overall best solution from a pool of complete solutions of the individual models, our combination approaches combine candidate arguments, or incomplete solutions, from different individual models. We show that our approach has better potential, i.e., the upper limit on the $\mathrm{F}_{1}$ score is higher and performance is better on several corpora.

A second novelty of this paper is that it performs a comparative analysis of several combination strategies for SRL, using the same framework -i.e., the same pool of candidates- and the same evaluation methodology. While a large number of combination approaches have been previously analyzed in the context of SRL or in the larger context of predicting structures in natural language texts -e.g., inference based on constraint satisfaction (Koomen, Punyakanok, Roth, \& Yih, 2005; Roth \& Yih, 2005), inference based in local learning (Màrquez et al., 2005), re-ranking (Collins, 2000; Haghighi et al., 2005) etc.it is still not clear which strategy performs best for semantic role labeling. In this paper we 
provide empirical answers to several important questions in this respect. For example, is a combination strategy based on constraint satisfaction better than an inference model based on learning? Or, how important is global feedback in the learning-based inference model? Our analysis indicates that the following issues are important traits of a state-of-the-art combination SRL system: (i) the individual models are combined at argument granularity rather than at the granularity of complete solutions (typical of re-ranking); (ii) the best combination strategy uses an inference model based in learning; and (iii) the learning-based inference benefits from max-margin classifiers and global feedback.

The paper is organized as follows. Section 2 introduces the semantic corpora used for training and evaluation. Section 3 overviews the proposed combination approaches. The individual SRL models are introduced in Section 4 and evaluated in Section 5. Section 6 lists the features used by the three combination models introduced in this paper. The combination models themselves are described in Section 7. Section 8 introduces an empirical analysis of the proposed combination methods. Section 9 reviews related work and Section 10 concludes the paper.

\section{Semantic Corpora}

In this paper we have used PropBank, an approximately one-million-word corpus annotated with predicate-argument structures (Palmer, Gildea, \& Kingsbury, 2005). To date, PropBank addresses only predicates lexicalized by verbs. Besides predicate-argument structures, PropBank contains full syntactic analysis of its sentences, because it extends the Wall Street Journal (WSJ) part of the Penn Treebank, a corpus that was previously annotated with syntactic information (Marcus, Santorini, \& Marcinkiewicz, 1994).

For any given predicate, a survey was carried out to determine the predicate usage, and, if required, the usages were divided into major senses. However, the senses are divided more on syntactic grounds than semantic, following the assumption that syntactic frames are a direct reflection of underlying semantics. The arguments of each predicate are numbered sequentially from A0 to A5. Generally, A0 stands for agent, A1 for theme or direct object, and A2 for indirect object, benefactive or instrument, but semantics tend to be verb specific. Additionally, predicates might have adjunctive arguments, referred to as AMs. For example, AM-LOC indicates a locative and AM-TMP indicates a temporal. Figure 1 shows a sample PropBank sentence where one predicate ("sold") has 4 arguments. Both regular and adjunctive arguments can be discontinuous, in which case the trailing argument fragments are prefixed by C-, e.g., "[A1 Both funds] are [predicate expected] [C-A1 to begin operation around March 1]." Finally, PropBank contains argument references (typically pronominal), which share the same label with the actual argument prefixed with $\mathrm{R}-{ }^{1}{ }^{1}$

In this paper we do not use any syntactic information from the Penn Treebank. Instead, we develop our models using automatically-generated syntax and named-entity (NE) labels, made available by the CoNLL-2005 shared task evaluation (Carreras \& Màrquez, 2005). From the CoNLL data, we use the syntactic trees generated by the Charniak parser (Char-

1. In the original PropBank annotations, co-referenced arguments appear as a single item, with no differentiation between the referent and the reference. Here we use the version of the data used in the CoNLL shared tasks, where reference arguments were automatically separated from their corresponding referents with simple pattern-matching rules. 
niak, 2000) to develop two individual models based on full syntactic analysis, and the chunk -i.e., basic syntactic phrase- labels and clause boundaries to construct a partial-syntax model. All individual models use the provided NE labels.

Switching from hand-corrected to automatically-generated syntactic information means that the PropBank assumption that each argument (or argument fragment for discontinuous arguments) maps to one syntactic phrase no longer holds, due to errors of the syntactic processors. Our analysis of the PropBank data indicates that only $91.36 \%$ of the semantic arguments can be matched to exactly one phrase generated by the Charniak parser. Essentially, this means that SRL approaches that make the assumption that each semantic argument maps to one syntactic construct can not recognize almost $9 \%$ of the arguments. The same statement can be made about approaches based on partial syntax with the caveat that in this setup arguments have to match a sequence of chunks. However, one expects that the degree of compatibility between syntactic chunks and semantic arguments is higher due to the finer granularity of the syntactic elements and because chunking algorithms perform better than full parsing algorithms. Indeed, our analysis of the same PropBank data supports this observation: $95.67 \%$ of the semantic arguments can be matched to a sequence of chunks generated by the CoNLL syntactic chunker.

Following the CoNLL-2005 setting we evaluated our system not only on PropBank but also on a fresh test set, derived from the Brown corpus. This second evaluation allows us to investigate the robustness of the proposed combination models.

\section{Overview of the Combination Strategies}

In this paper we introduce and analyze three combination strategies for the problem of semantic role labeling. The three combination strategies are implemented on a shared framework -detailed in Figure 2- which consists of several stages: (a) generation of candidate arguments, (b) candidate scoring, and finally (c) inference. For clarity, we describe first the proposed combination framework, i.e., the vertical flow in Figure 2. Then, we move to an overview of the three combination methodologies, shown horizontally in Figure 2.

In the candidate generation step, we merge the solutions of three individual SRL models into a unique pool of candidate arguments. The individual SRL models range from complete reliance on full parsing to using only partial syntactic information. For example, Model 1 is developed as a sequential tagger (using the B-I-O tagging scheme) with only partial syntactic information (basic phrases and clause boundaries), whereas Model 3 uses full syntactic analysis of the text and handles only arguments that map into exactly one syntactic constituent. We detail the individual SRL models in Section 4 and empirically evaluate them in Section 5.

In the candidate scoring phrase, we re-score all candidate arguments using both local information, e.g., the syntactic structure of the candidate argument, and global information, e.g., how many individual models have generated similar candidate arguments. We describe all the features used for candidate scoring in Section 6.

Finally, in the inference stage the combination models search for the best solution that is consistent with the domain constraints, e.g., two arguments for the same predicate cannot overlap or embed, a predicate may not have more than one core argument (A0-5), etc. 


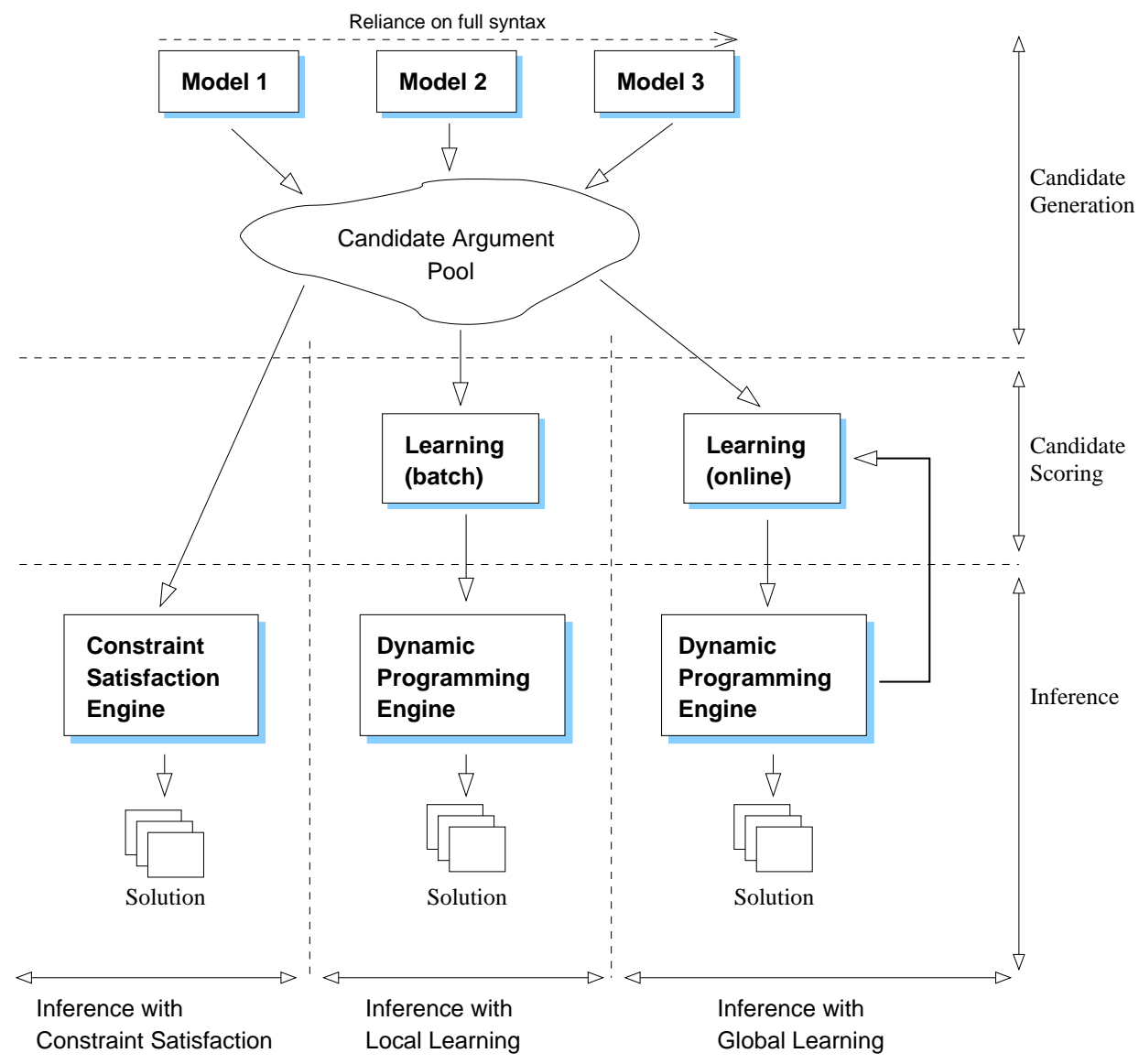

Figure 2: Overview of the proposed combination strategies.

All the combination approaches proposed in this paper share the same candidate argument pool. This guarantees that the results obtained by the different strategies on the same corpus are comparable. On the other hand, even though the candidate generation step is shared, the three combination methodologies differ significantly in their scoring and inference models.

The first combination strategy analyzed, inference with constraint satisfaction, skips the candidate scoring step completely and uses instead the probabilities output by the individual SRL models for each candidate argument. If the individual models' raw activations are not actual probabilities we convert them to probabilities using the softmax function (Bishop, 1995), before passing them to the inference component. The inference is implemented using a Constraint Satisfaction model that searches for the solution that maximizes a certain compatibility function. The compatibility function models not only the probability of the global solution but also the consistency of the solution according to the domain constraints. This combination strategy is based on the technique presented by Koomen et al. (2005). The main difference between the two systems is in the candidate generation step: we use three independent individual SRL models, whereas Komen et al. used the same SRL model 
trained on different syntactic views of the data, i.e., the top parse trees generated by the Charniak and Collins parsers (Charniak, 2000; Collins, 1999). Furthermore, we take our argument candidates from the set of complete solutions generated by the individual models, whereas Komen et al. take them from different syntactic trees, before constructing any complete solution. The obvious advantage of the inference model with Constraint Satisfaction is that it is unsupervised: no learning is necessary for candidate scoring, because the scores of the individual models are used. On the other hand, the Constraint Satisfaction model requires that the individual models provide raw activations, and, moreover, that the raw activations be convertible to true probabilities.

The second combination strategy proposed in this article, inference with local learning, re-scores all candidates in the pool using a set of binary discriminative classifiers. The classifiers assign to each argument a score measuring the confidence that the argument is part of the correct, global solution. The classifiers are trained in batch mode and are completely decoupled from the inference module. The inference component is implemented using a CKY-based dynamic programming algorithm (Younger, 1967). The main advantage of this strategy is that candidates are re-scored using significantly more information than what is available to each individual model. For example, we incorporate features that count the number of individual systems that generated the given candidate argument, several types of overlaps with candidate arguments of the same predicate and also with arguments of other predicates, structural information based on both full and partial syntax, etc. We describe the rich feature set used for the scoring of candidate arguments in Section 6. Also, this combination approach does not depend on the argument probabilities of the individual SRL models (but can incorporate them as features, if available). This combination approach is more complex than the previous strategy because it has an additional step that requires supervised learning: candidate scoring. Nevertheless, this does not mean that additional corpus is necessary: using cross validation, the candidate scoring classifiers can be trained on the same corpus used to train the individual SRL models. Moreover, we show in Section 8 that we obtain excellent performance even when the candidate scoring classifiers are trained on significantly less data than the individual SRL models.

Finally, the inference strategy with global learning investigates the contribution of global information to the inference model based on learning. This strategy incorporates global information in the previous inference model in two ways. First and most importantly, candidate scoring is now trained online with global feedback from the inference component. In other words, the online learning algorithm corrects the mistakes found when comparing the correct solution with the one generated after inference. Second, we integrate global information in the actual inference component: instead of performing inference for each proposition independently, we now do it for the whole sentence at once. This allows implementation of additional global domain constraints, e.g., arguments attached to different predicates can not overlap.

All the combination strategies proposed are described in detail in Section 7 and evaluated in Section 8. 


\section{Individual SRL Models}

This section introduces the three individual SRL models used by all the combination strategies discussed in this paper. The first two models are variations of the same algorithm: they both model the SRL problem as a sequential tagging task, where each semantic argument is matched to a sequence of non-embedding phrases, but Model 1 uses only partial syntax (chunks and clause boundaries), whereas Model 2 uses full syntax. The third model takes a more "traditional" approach by assuming that there exists a one-to-one mapping between semantic arguments and syntactic phrases.

It is important to note that all the combination strategies introduced later in the paper are independent of the individual SRL models used. In fact, in Section 8 we describe experiments that use not only these individual models but also the best performing SRL systems at the CoNLL-2005 evaluation (Carreras \& Màrquez, 2005). Nevertheless, we choose to focus mainly on the individual SRL approaches presented in this section for completeness and to show that state-of-the-art performance is possible with relatively simple SRL models.

\subsection{Models 1 and 2}

These models approach SRL as a sequential tagging task. In a pre-processing step, the input syntactic structures are traversed in order to select a subset of constituents organized sequentially (i.e., non embedding). The output of this process is a sequential tokenization of the input sentence for each of the verb predicates. Labeling these tokens with appropriate tags allows us to codify the complete argument structure of each predicate in the sentence.

More precisely, given a verb predicate, the sequential tokens are selected as follows: First, the input sentence is split into disjoint sequential segments using as markers for segment start/end the verb position and the boundaries of all the clauses that include the corresponding predicate constituent. Second, for each segment, the set of top-most non-overlapping syntactic constituents completely falling inside the segment are selected as tokens. Finally, these tokens are labeled with B-I-O tags, depending if they are at the beginning, inside, or outside of a predicate argument. Note that this strategy provides a set of sequential tokens covering the complete sentence. Also, it is independent of the syntactic annotation explored, assuming it provides clause boundaries.

Consider the example in Figure 3, which depicts the PropBank annotation of two verb predicates of a sentence ("release" and "hope") and the corresponding partial and full parse trees. Since both verbs are in the main clause of the sentence, only two segments of the sentence are considered for both predicates, i.e., those defining the left and right contexts of the verbs $\left(\left[\mathrm{w}_{1}\right.\right.$ :Others, $\ldots, \mathrm{w}_{3}:$ just $]$ and $\left[\mathrm{w}_{5}\right.$ :from, $\ldots, \mathrm{w}_{20}$ :big-time $]$ for predicate "release", and $\left[\mathrm{w}_{1}:\right.$ Others, ..., $\mathrm{w}_{8}:$ :] and $\left[\mathrm{w}_{10}:\right.$ the $, \ldots, \mathrm{w}_{20}$ :big-time $]$ for the predicate "hope"). Figure 4 shows the resulting tokenization for both predicates and the two alternative syntactic structures. In this case, the correct argument annotation can be recovered in all cases, assuming perfect labeling of the tokens.

It is worth noting that the resulting number of tokens to annotate is much lower than the number of words in all cases. Also, the codifications coming from full parsing have substantially fewer tokens than those coming from partial parsing. For example, for the predicate "hope", the difference in number of tokens between the two syntactic views is 


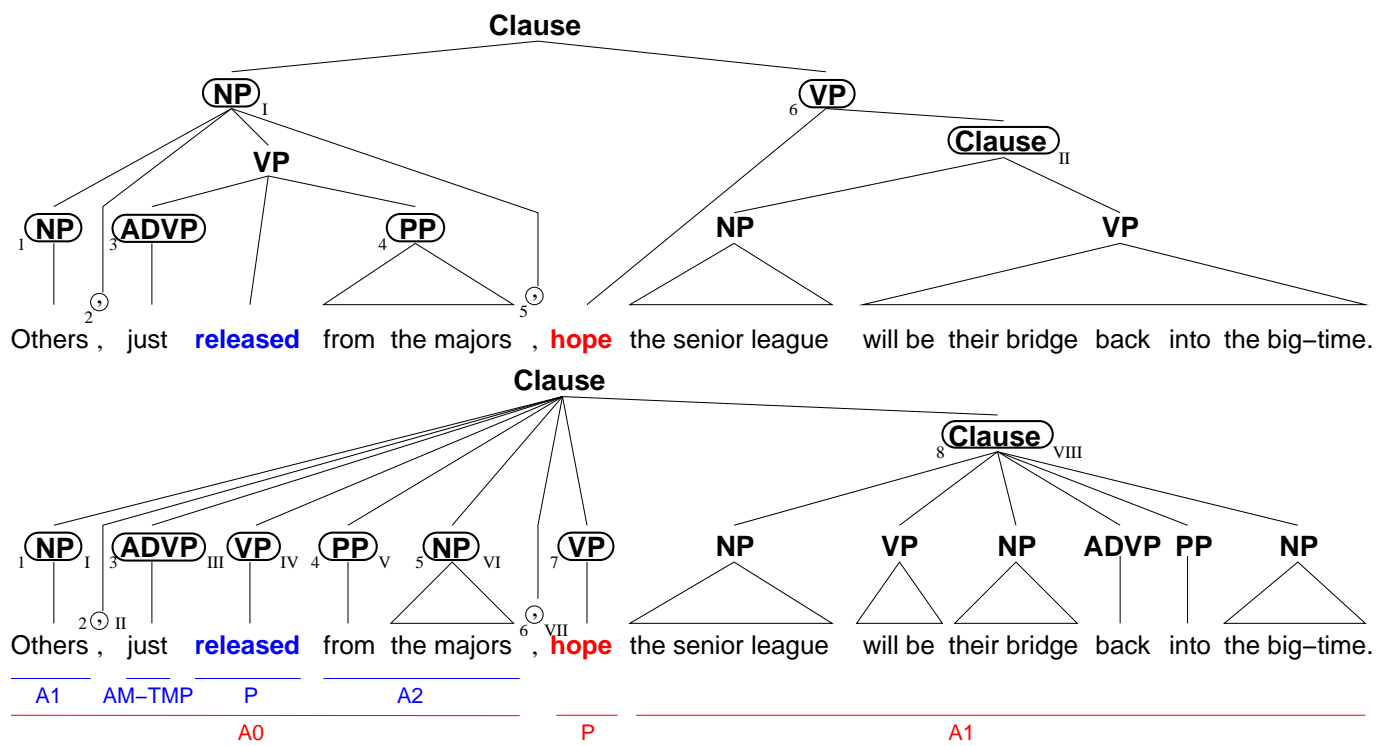

Figure 3: Annotation of an example sentence with two alternative syntactic structures. The lower tree corresponds to a partial parsing annotation (PP) with base chunks and clause structure, while the upper represents a full parse tree (FP). Semantic roles for two predicates ("release" and "hope") are also provided for the sentence. The encircled nodes in both trees correspond to the selected nodes by the process of sequential tokenization of the sentence. We mark the selected nodes for the predicate "release" with Western numerals and the nodes selected for "hope" with Roman numerals. See Figure 4 for more details.

particularly large ( 8 vs. 2 tokens). Obviously, the coarser the token granularity, the easier the problem of assigning correct output labelings (i.e., there are less tokens to label and also the long-distance relations among sentence constituents can be better captured). On the other hand, a coarser granularity tends to introduce more unrecoverable errors in the pre-processing stage. There is a clear trade-off, which is difficult to solve in advance. By using the two models in a combination scheme we can take advantage of the diverse sentence tokenizations (see Sections 7 and 8).

Compared to the more common tree node labeling approaches (e.g., the following Model 3), the B-I-O annotation of tokens has the advantage of permitting to correctly annotate some arguments that do not match a unique syntactic constituent. On the bad side, the heuristic pre-selection of only some candidate nodes for each predicate, i.e., the nodes that sequentially cover the sentence, makes the number of unrecoverable errors higher. Another source of errors common to all strategies are the errors introduced by real partial/full parsers. We have calculated that due to syntactic errors introduced in the pre-processing stage, the upper-bound recall figures are $95.67 \%$ for Model 1 and $90.32 \%$ for Model 2 using the datasets defined in Section 8 . 


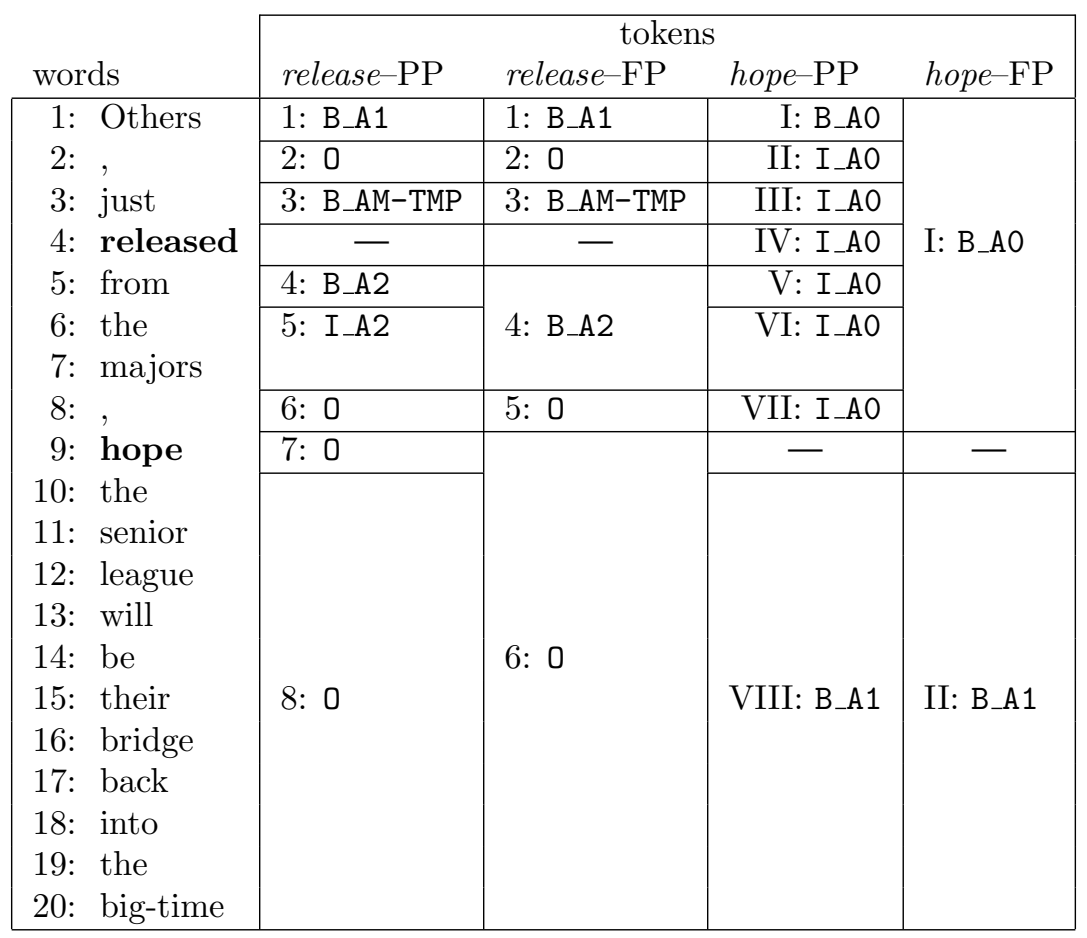

Figure 4: Sequential tokenization of the sentence in Figure 3 according to the two syntactic views and predicates (PP stands for partial parsing and FP for full parsing). The sentence and semantic role annotations are vertically displayed. Each token is numbered with the indexes that appear in the tree nodes of Figure 3 and contains the B-I-O annotation needed to codify the proper semantic role structure.

Approaching SRL as a sequential tagging task is not new. Hacioglu, Pradhan, Ward, Martin, and Jurafsky (2004) presented a system based on sequential tagging of base chunks with B-I-O labels, which was the best performing SRL system at the CoNLL-2004 shared task (Carreras \& Màrquez, 2004). The novelty of our approach resides in the fact that the sequence of syntactic tokens to label is extracted from a hierarchical syntactic annotation (either a partial or a full parse tree) and it is not restricted to base chunks (i.e., a token may correspond to a complex syntactic phrase or even a clause).

\subsubsection{FEATURES}

Once the tokens selected are labeled with B-I-O tags, they are converted into training examples by considering a rich set of features, mainly borrowed from state-of-the-art systems (Gildea \& Jurafsky, 2002; Carreras, Màrquez, \& Chrupała, 2004; Xue \& Palmer, 2004). These features codify properties from: (a) the focus token, (b) the target predicate, (c) the sentence fragment between the token and predicate, and (d) the dynamic context, i.e., B-I-O labels previously generated. We describe these four feature sets next. ${ }^{2}$

2. Features extracted from partial parsing and Named Entities are common to Model 1 and 2, while features coming from full parse trees only apply to Model 2. 


\section{Constituent structure features:}

- Constituent type and head: extracted using the head-word rules of Collins (1999). If the first element is a PP chunk, then the head of the first NP is extracted. For example, the type of the constituent "in the U.S." in Figure 1 is PP, but its head is "U.S." instead of "in".

- First and last words and POS tags of the constituent, e.g., "in" / IN and "U.S." /NNP for the constituent "in the U.S." in Figure 1.

- POS sequence: if it is less than 5 tags long, e.g., IN-DT-NNP for the above sample constituent.

- 2/3/4-grams of the POS sequence.

- Bag-of-words of nouns, adjectives, and adverbs. For example, the bag-of-nouns for the constituent "The luxury auto maker" is \{ "luxury", "auto", "maker"

- TOP sequence: sequence of types of the top-most syntactic elements in the constituent (if it is less than 5 elements long). In the case of full parsing this corresponds to the right-hand side of the rule expanding the constituent node. For example, the TOP sequence for the constituent "in the U.S." is IN-NP.

- 2/3/4-grams of the TOP sequence.

- Governing category as described by Gildea and Jurafsky (2002), which indicates if NP arguments are dominated by a sentence (typical for subjects) or a verb phrase (typical for objects). For example, the governing category for the constituent " 1,214 cars" in Figure 1 is VP, which hints that its corresponding semantic role will be object.

- NamedEntity, indicating if the constituent embeds or strictly matches a named entity along with its type. For example, the constituent "in the U.S." embeds a locative named entity: "U.S.".

- TMP, indicating if the constituent embeds or strictly matches a temporal keyword (automatically extracted from AM-TMP arguments of the training set). Among the most common temporal cue words extracted are: "year", "yesterday", "week", "month", etc. We used a total of 109 cue words.

- Previous and following words and POS tag of the constituent. For example, the previous word for the constituent "last year" in Figure 1 is "maker" /NN, and the next one is "sold"/VBD.

- The same features characterizing focus constituents are extracted for the two previous and following tokens, provided they are inside the boundaries of the current segment.

\section{Predicate structure features:}

- Predicate form, lemma, and POS tag, e.g., "sold", "sell", and VBD for the predicate in Figure 1.

- Chunk type and cardinality of verb phrase in which verb is included: single-word or multi-word. For example, the predicate in Figure 1 is included in a single-word VP chunk. 
- The predicate voice. We distinguish five voice types: active, passive, copulative, infinitive, and progressive.

- Binary flag indicating if the verb is a start/end of a clause.

- Sub-categorization rule, i.e., the phrase structure rule that expands the predicate's immediate parent, e.g., $\mathrm{S} \rightarrow$ NP NP VP for the predicate in Figure 1.

\section{Predicate-constituent features:}

- Relative position, distance in words and chunks, and level of embedding (in number of clause-levels) with respect to the constituent. For example, the constituent "in the U.S." in Figure 1 appears after the predicate, at a distance of 2 words or 1 chunk, and its level of embedding is 0 .

- Constituent path as described by Gildea and Jurafsky (2002) and all 3/4/5-grams of path constituents beginning at the verb predicate or ending at the constituent. For example, the syntactic path between the constituent "The luxury auto maker" and the predicate "sold" in Figure 1 is NP $\uparrow \mathrm{S} \downarrow$ VP $\downarrow$ VBD.

- Partial parsing path as described by Carreras et al. (2004) and all 3/4/5-grams of path elements beginning at the verb predicate or ending at the constituent. For example, the path NP + PP + NP + S $\downarrow$ VP $\downarrow$ VBD indicates that from the current NP token to the predicate there are $\mathrm{PP}, \mathrm{NP}$, and $\mathrm{S}$ constituents to the right (positive sign) at the same level of the token and then the path descends through the clause and a VP to find the predicate. The difference from the previous constituent path is that we do not have up arrows anymore but we introduce "horizontal" (left/right) movements at the same syntactic level.

- Syntactic frame as described by Xue and Palmer (2004). The syntactic frame captures the overall sentence structure using the predicate and the constituent as pivots. For example, the syntactic frame for the predicate "sold" and the constituent "in the U.S." is NP-NP- VP-NP-PP, with the current predicate and constituent emphasized. Knowing that there are other noun phrases before the predicate lowers the probability that this constituent serves as an agent (or A0).

\section{Dynamic features:}

- BIO-tag of the previous token. When training, the correct labels of the left context are used. When testing, this feature is dynamically codified as the tag previously assigned by the SRL tagger.

\subsubsection{Learning Algorithm and Sequence Tagging}

We used generalized AdaBoost with real-valued weak classifiers (Schapire \& Singer, 1999) as the base learning algorithm. Our version of the algorithm learns fixed-depth small decision trees as weak rules, which are then combined in the ensemble constructed by AdaBoost. We implemented a simple one-vs-all decomposition to address multi-class classification. In this way, a separate binary classifier has to be learned for each B-X and I-X argument label plus an extra classifier for the 0 decision. 
AdaBoost binary classifiers are then used for labeling test sequences, from left to right, using a recurrent sliding window approach with information about the tags assigned to the preceding tokens. As explained in the previous list of features, left tags already assigned are dynamically codified as features. Empirically, we found that the optimal left context to be taken into account reduces to only the previous token.

We tested two different tagging procedures. First, a greedy left-to-right assignment of the best scored label for each token. Second, a Viterbi search of the label sequence that maximizes the probability of the complete sequence. In this case, the classifiers' predictions were converted into probabilities using the softmax function described in Section 7.1. No significant improvements were obtained from the latter. We selected the former, which is faster, as our basic tagging algorithm for the experiments.

Finally, this tagging model enforces three basic constraints: (a) the B-I-O output labeling must codify a correct structure; (b) arguments cannot overlap with clause nor chunk boundaries; and (c) for each verb, A0-5 arguments not present in PropBank frames (taking the union of all rolesets for the different verb senses) are not considered.

\subsection{Model 3}

The third individual SRL model makes the strong assumption that each predicate argument maps to one syntactic constituent. For example, in Figure 1 A0 maps to a noun phrase, AM-LOC maps to a prepositional phrase, etc. This assumption holds well on hand-corrected parse trees and simplifies significantly the SRL process because only one syntactic constituent has to be correctly classified in order to recognize one semantic argument. On the other hand, this approach is limited when using automatically-generated syntactic trees. For example, only $91.36 \%$ of the arguments can be mapped to one of the syntactic constituents produced by the Charniak parser.

Using a bottom-up approach, Model 3 maps each argument to the first syntactic constituent that has the exact same boundaries and then climbs as high as possible in the tree across unary production chains. We currently ignore all arguments that do not map to a single syntactic constituent. The argument-constituent mapping is performed on the training set as preprocessing step. Figure 1 shows a mapping example between the semantic arguments of one verb and the corresponding sentence syntactic structure.

Once the mapping process completes, Model 3 extracts a rich set of lexical, syntactic,

and semantic features. Most of these features are inspired from previous work in parsing and SRL (Collins, 1999; Gildea \& Jurafsky, 2002; Surdeanu et al., 2003; Pradhan et al., 2005a). We describe the complete feature set implemented in Model 3 next.

\subsubsection{FeAtures}

Similarly to Models 1 and 2 we group the features in three categories, based on the properties they codify: (a) the argument constituent, (b) the target predicate, and (c) the relation between the constituent and predicate syntactic constituents.

\section{Constituent structure features:}

- The syntactic label of the candidate constituent.

- The constituent head word, suffixes of length 2, 3, and 4, lemma, and POS tag. 
- The constituent content word, suffixes of length 2, 3, and 4, lemma, POS tag, and NE label. Content words, which add informative lexicalized information different from the head word, were detected using the heuristics of Surdeanu et al. (2003). For example, the head word of the verb phrase "had placed" is the auxiliary verb "had", whereas the content word is "placed". Similarly, the content word of prepositional phrases is not the preposition itself (which is selected as the head word), but rather the head word of the attached phrase, e.g., "U.S." for the prepositional phrase "in the U.S.".

- The first and last constituent words and their POS tags.

- NE labels included in the candidate phrase.

- Binary features to indicate the presence of temporal cue words, i.e., words that appear often in AM-TMP phrases in training. We used the same list of temporal cue words as Models 1 and 2 .

- For each Treebank syntactic label we added a feature to indicate the number of such labels included in the candidate phrase.

- The TOP sequence of the constituent (constructed similarly to Model 2).

- The phrase label, head word and POS tag of the constituent parent, left sibling, and right sibling.

\section{Predicate structure features:}

- The predicate word and lemma.

- The predicate voice. Same definition as Models 1 and 2.

- A binary feature to indicate if the predicate is frequent (i.e., it appears more than twice in the training data) or not.

- Sub-categorization rule. Same definition as Models 1 and 2.

\section{Predicate-constituent features:}

- The path in the syntactic tree between the argument phrase and the predicate as a chain of syntactic labels along with the traversal direction (up or down). It is computed similarly to Model 2.

- The length of the above syntactic path.

- The number of clauses ( $\mathrm{S} *$ phrases) in the path. We store the overall clause count and also the number of clauses in the ascending and descending part of the path.

- The number of verb phrases (VP) in the path. Similarly to the above feature, we store three numbers: overall verb count, and the verb count in the ascending/descending part of the path.

- Generalized syntactic paths. We generalize the path in the syntactic tree, when it appears with more than 3 elements, using two templates: (a) Arg $\uparrow$ Ancestor $\downarrow N_{i} \downarrow$ Pred, where Arg is the argument label, Pred is the predicate label, Ancestor is the label of the common ancestor, and $\mathrm{N}_{i}$ is instantiated with each of the labels between 
Pred and Ancestor in the full path; and (b) Arg $\uparrow \mathrm{N}_{i} \uparrow$ Ancestor $\downarrow$ Pred, where $\mathrm{N}_{i}$ is instantiated with each of the labels between Arg and Ancestor in the full path. For example, in the path NP $\uparrow \mathrm{S} \downarrow$ VP $\downarrow$ SBAR $\downarrow \mathrm{S} \downarrow$ VP the argument label is the first NP, the predicate label is the last VP, and the common ancestor's label is the first S. Hence, using the last template, this path is generalized to the following three features: NP $\uparrow$ $\mathrm{S} \downarrow \mathrm{VP} \downarrow \mathrm{VP}, \mathrm{NP} \uparrow \mathrm{S} \downarrow \mathrm{SBAR} \downarrow \mathrm{VP}$, and NP $\uparrow \mathrm{S} \downarrow \mathrm{S} \downarrow$ VP. This generalization reduces the sparsity of the complete constituent-predicate path feature using a different strategy than Models 1 and 2, which implement a $n$-gram based approach.

- The subsumption count, i.e., the difference between the depths in the syntactic tree of the argument and predicate constituents. This value is 0 if the two phrases share the same parent.

- The governing category, similar to Models 1 and 2.

- The surface distance between the predicate and the argument phrases encoded as: the number of tokens, verb terminals (VB*), commas, and coordinations (CC) between the argument and predicate phrases, and a binary feature to indicate if the two constituents are adjacent. For example, the surface distance between the argument candidate "Others" and the predicate "hope" in the Figure 3 example: "Others, just released from the majors, hope the senior league..." is 7 tokens, 1 verb, 2 commas, and 0 coordinations. These features, originally proposed by Collins (1999) for his dependency parsing model, capture robust, syntax-independent information about the sentence structure. For example, a constituent is unlikely to be the argument of a verb if another verb appears between the two phrases.

- A binary feature to indicate if the argument starts with a predicate particle, i.e., a token seen with the RP* POS tag and directly attached to the predicate in training. The motivation for this feature is to avoid the inclusion of predicate particles in the argument constituent. For example, without this feature, a SRL system will tend to incorrectly include the predicate particle in the argument for the text: "take [A1 over the organization]", because the marked text is commonly incorrectly parsed as a prepositional phrase and a large number of prepositional phrases directly attached to a verb are arguments for the corresponding predicate.

\subsubsection{Classifier}

Similarly to Models 1 and 2, Model 3 trains one-vs-all classifiers using AdaBoost for the most common argument labels. To reduce the sample space, Model 3 selects training examples (both positive and negative) only from: (a) the first clause that includes the predicate, or (b) from phrases that appear to the left of the predicate in the sentence. More than $98 \%$ of the argument constituents fall into one of these classes.

At prediction time the classifiers are combined using a simple greedy technique that iteratively assigns to each predicate the argument classified with the highest confidence. For each predicate we consider as candidates all AM attributes, but only numbered attributes indicated in the corresponding PropBank frame. Additionally, this greedy strategy enforces a limited number of domain knowledge constraints in the generated solution: (a) arguments can not overlap in any form, (b) no duplicate arguments are allowed for A0-5, and (c) each 
predicate can have numbered arguments, i.e., A0-5, only from the subset present in its PropBank frame. These constraints are somewhat different from the constraints used by Models 1 and 2: (i) Model 3 does not use the B-I-O representation hence the constraint that the B-I-O labeling be correct does not apply; and (ii) Models 1 and 2 do not enforce the constraint that numbered arguments can not be duplicated because its implementation is not straightforward in this architecture.

\section{Performance of the Individual Models}

In this section we analyze the performance of the three individual SRL models proposed. Our three SRL systems were trained using the complete CoNLL-2005 training set (PropBank/Treebank sections 2 to 21). To avoid the overfitting of the syntactic processors -i.e., part-of-speech tagger, chunker, and Charniak's full parser- we partitioned the PropBank training set into five folds and for each fold we used the output of the syntactic processors that were trained on the other four folds. The models were tuned on a separate development partition (Treebank section 24) and evaluated on two corpora: (a) Treebank section 23, which consists of Wall Street Journal (WSJ) documents, and (b) on three sections of the Brown corpus, semantically annotated by the PropBank team for the CoNLL-2005 shared task evaluation.

All the classifiers for our individual models were developed using AdaBoost with decision trees of depth 4 (i.e., each branch may represent a conjunction of at most 4 basic features). Each classification model was trained for up to 2,000 rounds. We applied some simplifications to keep training times and memory requirements inside admissible bounds: (a) we have trained only the most frequent argument labels: top 41 for Model 1, top 35 for Model 2, and top 24 for Model 3; (b) we discarded all features occurring less than 15 times in the training set, and (c) for each Model 3 classifier, we have limited the number of negative training samples to the first 500,000 negative samples extracted in the PropBank traversal $^{3}$.

Table 1 summarizes the results of the three models on the WSJ and Brown corpora. We include the percentage of perfect propositions detected by each model ("PProps"), i.e., predicates recognized with all their arguments, the overall precision, recall, and $\mathrm{F}_{1}$ measure $^{4}$. The results summarized in Table 1 indicate that all individual systems have a solid performance. Although none of them would rank in the top 3 in the CoNLL2005 evaluation (Carreras \& Màrquez, 2005), their performance is comparable to the best individual systems presented at that evaluation exercise ${ }^{5}$. Consistently with other systems evaluated on the Brown corpus, all our models experience a severe performance drop in this corpus, due to the lower performance of the linguistic processors.

As expected, the models based on full parsing (2 and 3) perform better than the model based on partial syntax. But, interestingly, the difference is not large (e.g., less than 2 points

3. The distribution of samples for the Model 3 classifiers is very biased towards negative samples because, in the worst case, any syntactic constituent in the same sentence with the predicate is a potential argument.

4. The significance intervals for the $\mathrm{F}_{1}$ measure have been obtained using bootstrap resampling (Noreen, 1989). $F_{1}$ rates outside of these intervals are assumed to be significantly different from the related $F_{1}$ rate $(p<0.05)$.

5. The best performing SRL systems at CoNLL were a combination of several subsystems. See section 9 for details. 


\begin{tabular}{|c|c|c|c|c|}
\hline WSJ & PProps & Precision & Recall & $\mathrm{F}_{1}$ \\
\hline Model 1 & $48.45 \%$ & $78.76 \%$ & $72.44 \%$ & $75.47 \pm 0.8$ \\
\hline Model 2 & $52.04 \%$ & $79.65 \%$ & $74.92 \%$ & $\mathbf{7 7 . 2 1} \pm 0.8$ \\
\hline Model 3 & $45.28 \%$ & $80.32 \%$ & $72.95 \%$ & $76.46 \pm 0.6$ \\
\hline \multicolumn{5}{|l|}{ Brown } \\
\hline Model 1 & $30.85 \%$ & $67.72 \%$ & $58.29 \%$ & $62.65 \pm 2.1$ \\
\hline Model 2 & $36.44 \%$ & $71.82 \%$ & $\mathbf{6 4 . 0 3} \%$ & $\mathbf{6 7 . 7 0} \pm 1.9$ \\
\hline Model 3 & $29.48 \%$ & $72.41 \%$ & $59.67 \%$ & $65.42 \pm 2.1$ \\
\hline
\end{tabular}

Table 1: Overall results of the individual models in the WSJ and Brown test sets.

\begin{tabular}{|c|c|c|c|c|c|}
\cline { 2 - 6 } \multicolumn{1}{c|}{} & $\mathrm{A} 0$ & $\mathrm{~A} 1$ & $\mathrm{~A} 2$ & $\mathrm{~A} 3$ & $\mathrm{~A} 4$ \\
\hline Model 1 $\mathrm{F}_{1}$ & 83.37 & 75.13 & $\mathbf{6 7 . 3 3}$ & 61.92 & 72.73 \\
Model 2 $\mathrm{F}_{1}$ & $\mathbf{8 6 . 6 5}$ & $\mathbf{7 7 . 0 6}$ & 65.04 & 62.72 & 72.43 \\
Model 3 $\mathrm{F}_{1}$ & 86.14 & 75.83 & 65.55 & $\mathbf{6 5 . 2 6}$ & $\mathbf{7 3 . 8 5}$ \\
\hline
\end{tabular}

Table 2: $\mathrm{F}_{1}$ scores of the individual systems for the A0-4 arguments in the WSJ test.

in $\mathrm{F}_{1}$ in the WSJ corpus), evincing that having base syntactic chunks and clause boundaries is enough to obtain competitive performance. More importantly, the full-parsing models are not always better than the partial-syntax model. Table 2 lists the $\mathrm{F}_{1}$ measure for the three models for the first five numbered arguments. Table 2 shows that Model 2, our overall best performing individual system, achieves the best F-measure for A0 and A1 (typically subjects and direct objects), but Model 1, the partial-syntax model, performs best for the A2 (typically indirect objects, instruments, or benefactives). The explanation for this behavior is that indirect objects tend to be farther from their predicates and accumulate more parsing errors. From the models based on full syntax, Model 2 has better recall whereas Model 3 has better precision, because Model 3 filters out all candidate arguments that do not match a single syntactic constituent. Generally, Table 2 shows that all models have strong and weak points. This is further justification for our focus on combination strategies that combine several independent models.

\section{Features of the Combination Models}

As detailed in Section 3, in this paper we analyze two classes of combination strategies for the problem of semantic role labeling: (a) an inference model with constraint satisfaction, which finds the set of candidate arguments that maximizes a global cost function, and (b) two inference strategies based on learning, where candidates are scored and ranked using discriminative classifiers. From the perspective of the feature space, the main difference between these two types of combination models is that the input of the first combination strategy is limited to the argument probabilities produced by the individual systems, whereas the last class of combination approaches incorporates a much larger feature set in their ranking classifiers. For robustness, in this paper we use only features that are extracted from the solutions provided by the individual systems, hence are independent of the 


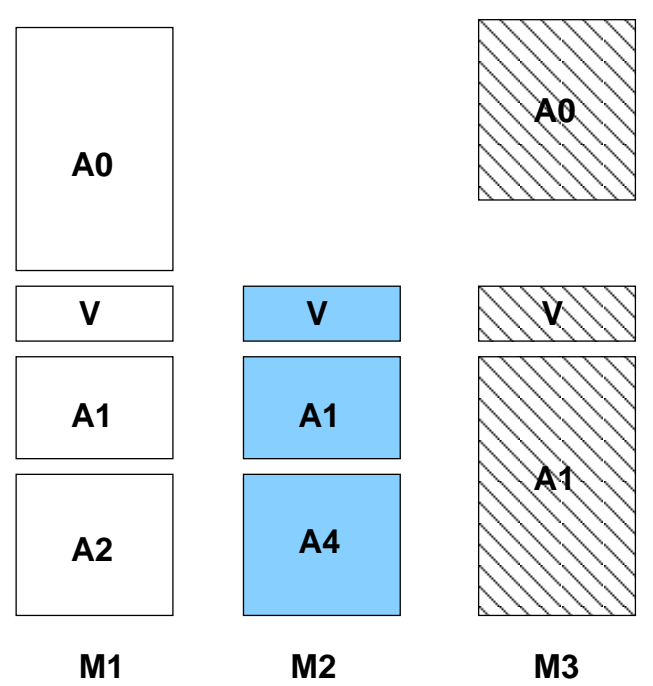

Figure 5: Sample solutions proposed for the same predicate by three individual SRL models: M1, M2 and M3. Argument candidates are displayed vertically for each system.

individual models ${ }^{6}$. We describe all these features next. All examples given in this section are based on Figures 5 and 6 .

Voting features - these features quantify the votes received by each argument from the individual systems. This set includes the following features:

- The label of the candidate argument, e.g., A0 for the first argument proposed by system M1 in Figure 5.

- The number of systems that generated an argument with this label and span. For the example shown in Figure 5, this feature has value 1 for the argument A0 proposed by M1 and 2 for M1's A1, because system M2 proposed the same argument.

- The unique ids of all the systems that generated an argument with this label and span, e.g., M1 and M2 for the argument A1 proposed by M1 or M2 in Figure 5.

- The argument sequence for this predicate for all the systems that generated an argument with this label and span. For example, the argument sequence generated by system M1 for the proposition illustrated in Figure 5 is: A0 - V - A1 - A2. This feature attempts to capture information at proposition level, e.g., a combination model might learn to trust model M1 more for the argument sequence A0 - V - A1 - A2, M2 for another sequence, etc.

Same-predicate overlap features - these features measure the overlap between different arguments produced by the individual SRL models for the same predicate:

6. With the exception of the argument probabilities, which are required by the constraint satisfaction model. 
- The number and unique ids of all the systems that generated an argument with the same span but different label. For the example shown in Figure 5, these features have values 1 and M2 for the argument A2 proposed by M1, because model M2 proposed argument A4 with the same span.

- The number and unique ids of all the systems that generated an argument included in the current argument. For the candidate argument A0 proposed by model M1 in Figure 5, these features have values 1 and M3, because M3 generated argument A0, which is included in M1's A0.

- In the same spirit, we generate the number and unique ids of all the systems that generated an argument that contains the current argument, and the number and unique $i d s$ of all the systems that generated an argument that overlaps - but does not include nor contain - the current argument.

Other-predicate overlap features - these features quantify the overlap between different arguments produced by the individual SRL models for other predicates. We generate the same features as the previous feature group, with the difference that we now compare arguments generated for different predicates. The motivation for these overlap features is that, according to the PropBank annotations, no form of overlap is allowed among arguments attached to the same predicate, and only inclusion or containment is permitted between arguments assigned to different predicates. The overlap features are meant to detect when these domain constraints are not satisfied by a candidate argument, which is an indication, if the evidence is strong, that the candidate is incorrect.

Partial-syntax features - these features codify the structure of the argument and the distance between the argument and the predicate using only partial syntactic information, i.e., chunks and clause boundaries (see Figure 6 for an example). Note that these features are inherently different from the features used by Model 1, because Model 1 evaluates each individual chunk part of a candidate argument, whereas here we codify properties of the complete argument constituent. We describe the partial-syntax features below.

- Length in tokens and chunks of the argument constituent, e.g., 4 and 1 for argument A0 in Figure 6.

- The sequence of chunks included in the argument constituent, e.g., PP NP for the argument AM-LOC in Figure 6. If the chunk sequence is too large, we store $n$-grams of length 10 for the start and end of the sequence.

- The sequence of clause boundaries, i.e., clause beginning or ending, included in the argument constituent.

- The named entity types included in the argument constituent, e.g., LOCATION for the AM-LOC argument in Figure 6.

- Position of the argument: before/after the predicate in the sentence, e.g., after for A1 in Figure 6.

- A Boolean flag to indicate if the argument constituent is adjacent to the predicate, e.g., false for $\mathrm{A} 0$ and true for $\mathrm{A} 1$ in Figure 6. 


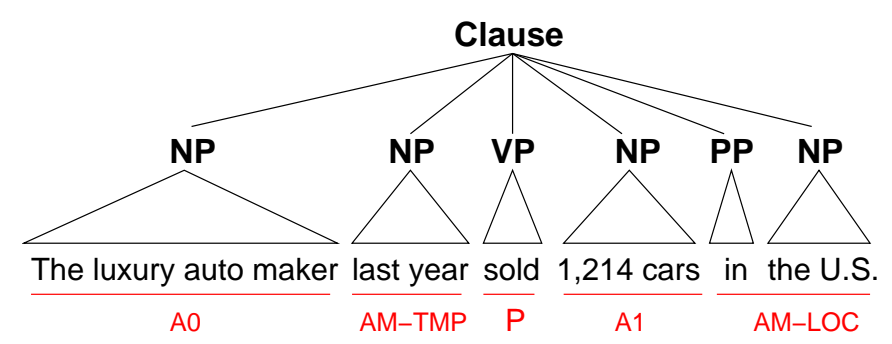

Figure 6: Sample proposition with partial syntactic information.

- The sequence of chunks between the argument constituent and the predicate, e.g., the chunk sequence between the predicate and the argument AM-LOC in Figure 6 is: NP. Similarly to the above chunk sequence feature, if the sequence is too large, we store starting and ending $n$-grams.

- The number of chunks between the predicate and the argument, e.g., 1 for AM-LOC in Figure 6.

- The sequence of clause boundaries between the argument constituent and the predicate.

- The clause subsumption count, i.e., the difference between the depths in the clause tree of the argument and predicate constituents. This value is 0 if the two phrases are included in the same clause.

Full-syntax features - these features codify the structure of the argument constituent, the predicate, and the distance between the two using full syntactic information. The full-syntax features are replicated from Model 3 (see Section 4.2), which assumes that a one-to-one mapping from semantic constituents to syntactic phrases exists. Unlike Model 3 which ignores arguments that can not be matched against a syntactic constituent, if such an exact mapping does not exist due to the inclusion of candidates from Models 1 and 2, we generate an approximate mapping from the unmapped semantic constituent to the largest phrase that is included in the given span and has the same left boundary as the semantic constituent. This heuristic guarantees that we capture at least some of the semantic constituents' syntactic structure.

The motivation for the partial and full-syntax features is to learn the "preferences" of the individual SRL models. For example, with these features a combination classifier might learn to trust model M1 for arguments that are closer than 3 chunks to the predicate, model M2 when the predicate-argument syntactic path is NP $\uparrow \mathrm{S} \downarrow$ VP $\downarrow$ SBAR $\downarrow S \downarrow$ VP, etc.

Individual systems' argument probabilities - each individual model outputs a confidence score for each of their proposed arguments. These scores are converted into probabilities using the softmax function as described in detail in Section 7.1. The combination strategy based on constraint satisfaction (Section 7.1) uses these probabilities as they are, while the other two strategies based on meta-learning (Section 7.2) have to discretize the probabilities to include them as features. To do so, each probability value is matched to 
one of five probability intervals and the corresponding interval is used as the feature. The probability intervals are dynamically constructed for each argument label and each individual system such that the corresponding system predictions for this argument label are uniformly distributed across the intervals.

In Section 8.4 we empirically analyze the contribution of each of these proposed feature sets to the performance of our best combination model.

\section{Combination Strategies}

In this section we detail the combination strategies proposed in this paper: (a) a combination model with constraint satisfaction, which aims at finding the set of candidate arguments that maximizes a global cost function, and (b) two combination models with inference based on learning, where candidates are scored and ranked using discriminative classifiers. In the previous section we described the complete feature set made available to all approaches. Here we focus on the machine learning paradigm deployed by each of the combination models.

\subsection{Inference with Constraint Satisfaction}

The Constraint Satisfaction model selects a subset of candidate arguments that maximizes a compatibility function subject to the fulfillment of a set of structural constraints that ensure consistency of the solution. The compatibility function is based on the probabilities given by individual SRL models to the candidate arguments. In this work we use Integer Linear Programming to solve the constraint satisfaction problem. This approach was first proposed by Roth and Yih (2004) and applied to semantic role labeling by Punyakanok, Roth, Yih, and Zimak (2004), Koomen et al. (2005), among others. We follow the setting of Komen et al., which is taken as a reference.

As a first step, the scores from each model are normalized into probabilities. The scores yielded by the classifiers are signed and unbounded real numbers, but experimental evidence shows that the confidence in the predictions (taken as the absolute value of the raw scores) correlates well with the classification accuracy. Thus, the softmax function (Bishop, 1995) is used to convert the set of unbounded scores into probabilities. If there are $k$ possible output labels for a given argument and $\operatorname{sco}\left(l_{i}\right)$ denotes the score of label $l_{i}$ output by a fixed SRL model, then the estimated probability for this label is:

$$
\hat{p}\left(l_{i}\right)=\frac{e^{\gamma \operatorname{sco}\left(l_{i}\right)}}{\sum_{j=1}^{k} e^{\gamma \operatorname{sco}\left(l_{j}\right)}}
$$

The $\gamma$ parameter of the above formula can be empirically adjusted to avoid overly skewed probability distributions and to normalize the scores of the three individual models to a similar range of values. See more details about our experimental setting in Section 8.1.

Candidate selection is performed via Integer Linear Programming (ILP). The program goal is to maximize a compatibility function modeling the global confidence of the selected set of candidates, subject to a set of linear constraints. All the variables involved in the task take integer values and may appear in first degree polynomials only.

An abstract ILP process can be described in a simple fashion as: given a set of variables $V=\left\{v_{1}, \ldots, v_{n}\right\}$, it aims to maximize the global compatibility of a label assignment 
$\left\{l_{1}, \ldots, l_{n}\right\}$ to these variables. A local compatibility function $c_{v}(l)$ defines the compatibility of assigning label $l$ to variable $v$. The global compatibility function $C\left(l_{1}, \ldots, l_{n}\right)$ is taken as the sum of each local assignment compatibility, so the goal of the ILP process can be written as:

$$
\underset{l_{1}, \ldots, l_{n}}{\operatorname{argmax}} C\left(l_{1}, \ldots, l_{n}\right)=\underset{l_{1}, \ldots, l_{n}}{\operatorname{argmax}} \sum_{i=1}^{n} c_{v_{i}}\left(l_{i}\right)
$$

where the constraints are described in a set of accompanying integer linear equations involving the variables of the problem.

If one wants to codify soft constraints instead of hard, there is the possibility of considering them as a penalty component in the compatibility function. In this case, each constraint $r \in R$ can be seen as a function which takes the current label assignment and outputs a real number, which is 0 when the constraint is satisfied and a positive number when not, indicating the penalty imposed to the compatibility function. The new expression of the compatibility function to maximize is:

$$
C\left(l_{1}, \ldots, l_{n}\right)=\sum_{i=1}^{n} c_{v_{i}}\left(l_{i}\right)-\sum_{r \in R} r\left(l_{1}, \ldots, l_{n}\right)
$$

Note that the hard constraints can also be simulated in this setting by making them output a very large positive number when they are violated.

In our particular problem, we have a binary-valued variable $v_{i}$ for each of the $N$ argument candidates generated by the SRL models, i.e., $l_{i}$ labels are in $\{0,1\}$. Given a label assignment, the arguments with $l_{i}=1$ are selected to form the solution, while the others (those where $l_{i}=0$ ) are filtered out. For each variable $v_{i}$, we also have the probability values, $p_{i j}$, calculated from the score of model $j$ on argument i, according to the softmax formula described above ${ }^{7}$. In a first approach, the compatibility function $c_{v}\left(l_{i}\right)$ equals to $\left(\sum_{j=1}^{M} p_{i j}\right) l_{i}$, where the number of models, $M$, is 3 in our case ${ }^{8}$.

Under this definition, maximizing the compatibility function is equivalent to maximizing the sum of the probabilities given by the models to the argument candidates considered in the solution. Since this function is always positive, the global score increases directly with the number of selected candidates. As a consequence, the model is biased towards the maximization of the number of candidates included in the solution (e.g., tending to select a lot of small non-overlapping arguments). Following Koomen et al. (2005), this bias can be corrected by adding a new score $o_{i}$, which sums to the compatibility function when the $i$-th candidate is not selected in the solution. The global compatibility function needs to be rewritten to encompass this new information. Formalized as an ILP equation, it looks like:

$$
\underset{L \in\{0,1\}^{N}}{\operatorname{argmax}} C\left(l_{1}, \ldots, l_{N}\right)=\underset{L \in\{0,1\}^{N}}{\operatorname{argmax}} \sum_{i=1}^{N}\left(\sum_{j=1}^{M} p_{i j}\right) l_{i}+o_{i}\left(1-l_{i}\right)
$$

7. If model $j$ does not propose argument $i$ then we consider $p_{i j}=0$.

8. Instead of accumulating the probabilities of all models for a given candidate argument, one could consider a different variable for each model prediction and introduce a constraint forcing all these variables to take the same value at the end of the optimization problem. The two alternatives are equivalent. 
where the constraints are expressed in separated integer linear equations. It is not possible to define a priori the value of $o_{i}$. Komen et al. used a validation corpus to empirically estimate a constant value for all $o_{i}$ (i.e., independent from the argument candidate) ${ }^{9}$. We will use exactly the same solution of working with a single constant value, to which we will refer as $O$.

Regarding the consistency constraints, we have considered the following six:

1. Two candidate arguments for the same verb can not overlap nor embed.

2. A verb may not have two core arguments with the same type label A0-A5.

3. If there is an argument $\mathrm{R}-\mathrm{X}$ for a verb, there has to be also an $\mathrm{X}$ argument for the same verb.

4. If there is an argument $\mathrm{C}-\mathrm{X}$ for a verb, there has to be also an $\mathrm{X}$ argument before the $\mathrm{C}-\mathrm{X}$ for the same verb.

5. Arguments from two different verbs can not overlap, but they can embed.

6. Two different verbs can not share the same AM-X, R-AM-X or C-X arguments.

Constraints 1-4 are also included in our reference work (Punyakanok et al., 2004). No other constraints from that paper need to be checked here since each individual model outputs only consistent solutions. Constraints 5 and 6 , which restrict the set of compatible arguments among different predicates in the sentence, are original to this work. In the Integer Linear Programming setting the constraints are written as inequalities. For example, if $A_{i}$ is the argument label of the $i$-th candidate and $V_{i}$ its verb predicate, constraint number 2 is written as: $\sum_{\left(A_{i}=a \wedge V_{i}=v\right)} l_{i} \leq 1$, for a given verb $v$ and argument label $a$. The other constraints have similar translations into inequalities.

Constraint satisfaction optimization will be applied in two different ways to obtain the complete output annotation of a sentence. In the first one, we proceed verb by verb independently to find their best selection of candidate arguments using only constraints 1 through 4. We call this approach local optimization. In the second scenario all the candidate arguments in the sentence are considered at once and constraints 1 through 6 are enforced. We will refer to this second strategy as global optimization. In both scenarios the compatibility function will be the same, but constraints need some rewriting in the global scenario because they have to include information about the concrete predicate.

In Section 8.3 we will extensively evaluate the presented inference model based on Constraint Satisfaction, and we will describe some experiments covering the following topics: (a) the contribution of each of the proposed constraints; (b) the performance of local vs. global optimization; and (c) the precision-recall tradeoff by varying the value of the bias-correction parameter.

\subsection{Inference Based On Learning}

This combination model consists of two stages: a candidate scoring phase, which scores candidate arguments in the pool using a series of discriminative classifiers, and an inference stage, which selects the best overall solution that is consistent with the domain constraints.

9. Instead of working with a constant, one could try to set the $o_{i}$ value for each candidate, taking into account some contextual features of the candidate. We plan to explore this option in the near future. 
The first and most important component of this combination strategy is the candidate scoring module, which assigns to each candidate argument a score equal to the confidence that this argument is part of the global solution. It is formed by discriminative functions, one for each role label. Below, we devise two different strategies to train the discriminative functions.

After scoring candidate arguments, the final global solution is built by the inference module, which looks for the best scored argument structure that satisfies the domain specific constraints. Here, a global solution is a subset of candidate arguments, and its score is defined as the sum of confidence values of the arguments that form it. We currently consider three constraints to determine which solutions are valid:

(a) Candidate arguments for the same predicate can not overlap nor embed.

(b) In a predicate, no duplicate arguments are allowed for the numbered arguments A0-5.

(c) Arguments of a predicate can be embedded within arguments of other predicates but they can not overlap.

The set of constraints can be extended with any other rules, but in our particular case, we know that some constraints, e.g., providing only arguments indicated in the corresponding PropBank frame, are already guaranteed by the individual models, and others, e.g., constraints 3 and 4 in the previous sub-section, have no positive impact on the overall performance (see Section 8.3 for the empirical analysis). The inference algorithm we use is a bottom-up CKY-based dynamic programming strategy (Younger, 1967). It builds the solution that maximizes the sum of argument confidences while satisfying the constraints, in cubic time.

Next, we describe two different strategies to train the functions that score candidate arguments. The first is a local strategy: each function is trained as a binary batch classifier, independently of the combination process which enforces the domain constraints. The second is a global strategy: functions are trained as online rankers, taking into account the interactions that take place during the combination process to decide between one argument or another.

In both training strategies, the discriminative functions employ the same representation of arguments, using the complete feature set described in Section 6 (we analyze the contribution of each feature group in Section 8). Our intuition was that the rich feature space introduced in Section 6 should allow the gathering of sufficient statistics for robust scoring of the candidate arguments. For example, the scoring classifiers might learn that a candidate is to be trusted if: (a) two individual systems proposed it, (b) if its label is A2 and it was generated by Model 1, or (c) if it was proposed by Model 2 within a certain argument sequence.

\subsubsection{Learning Local Classifiers}

This combination process follows a cascaded architecture, in which the learning component is decoupled from the inference module. In particular, the training strategy consists of training a binary classifier for each role label. The target of each label-based classifier is to determine whether a candidate argument actually belongs to the correct proposition of the corresponding predicate, and to output a confidence value for this decision. 
The specific training strategy is as follows. The training data consists of a pool of labeled candidate arguments (proposed by individual systems). Each candidate is either positive, in that it is actually a correct argument of some sentence, or negative, if it is not correct. The strategy trains a binary classifier for each role label $l$, independently of other labels. To do so, it concentrates on the candidate arguments of the data that have label $l$. This forms a dataset for binary classification, specific to the label $l$. With it, a binary classifier can be trained using any of the existing techniques for binary classification, with the only requirement that our combination strategy needs confidence values with each binary prediction. In Section 8 we provide experiments using SVMs to train such local classifiers.

In all, each classifier is trained independently of other classifiers and the inference module. Looking globally at the combination process, each classifier can be seen as an argument filtering component that decides which candidates are actual arguments using a much richer representation than the individual models. In this context, the inference engine is used as a conflict resolution engine, to ensure that the combined solutions are valid argument structures for sentences.

\subsubsection{Learning Global Rankers}

This combination process couples learning and inference, i.e., the scoring functions are trained to behave accurately within the inference module. In other words, the training strategy here is global: the target is to train a global function that maps a set of argument candidates for a sentence into a valid argument structure. In our setting, the global function is a composition of scoring functions - one for each label, same as the previous strategy. Unlike the previous strategy, which is completely decoupled from the inference engine, here the policy to map a set of candidates into a solution is that determined by the inference engine.

In recent years, research has been very active in global learning methods for tagging, parsing and, in general, structure prediction problems (Collins, 2002; Taskar, Guestrin, \& Koller, 2003; Taskar, Klein, Collins, Koller, \& Manning, 2004; Tsochantaridis, Hofmann, Joachims, \& Altun, 2004). In this article, we make use of the simplest technique for global learning: an online learning approach that uses Perceptron (Collins, 2002). The general idea of the algorithm is similar to the original Perceptron (Rosenblatt, 1958): correcting the mistakes of a linear predictor made while visiting training examples, in an additive manner. The key point for learning global rankers relies on the criteria that determines what is a mistake for the function being trained, an idea that has been exploited in a similar way in multiclass and ranking scenarios by Crammer and Singer (2003a, 2003b).

The Perceptron algorithm in our combination system works as follows (pseudocode of the algorithm is given in Figure 7 ). Let $1 \ldots L$ be the possible role labels, and let $\mathcal{W}=\left\{\mathbf{w}_{1} \ldots \mathbf{w}_{L}\right\}$ be the set of parameter vectors of the scoring functions, one for each label. Perceptron initializes the vectors in $\mathcal{W}$ to zero, and then proceeds to cycle through the training examples, visiting one at a time. In our case, a training example is a pair $(y, \mathcal{A})$, where $y$ is the correct solution of the example and $\mathcal{A}$ is the set of candidate arguments for it. Note that both $y$ and $\mathcal{A}$ are sets of labeled arguments, and thus we can make use of the set difference. We will note as $a$ a particular argument, as $l$ the label of $a$, and as 


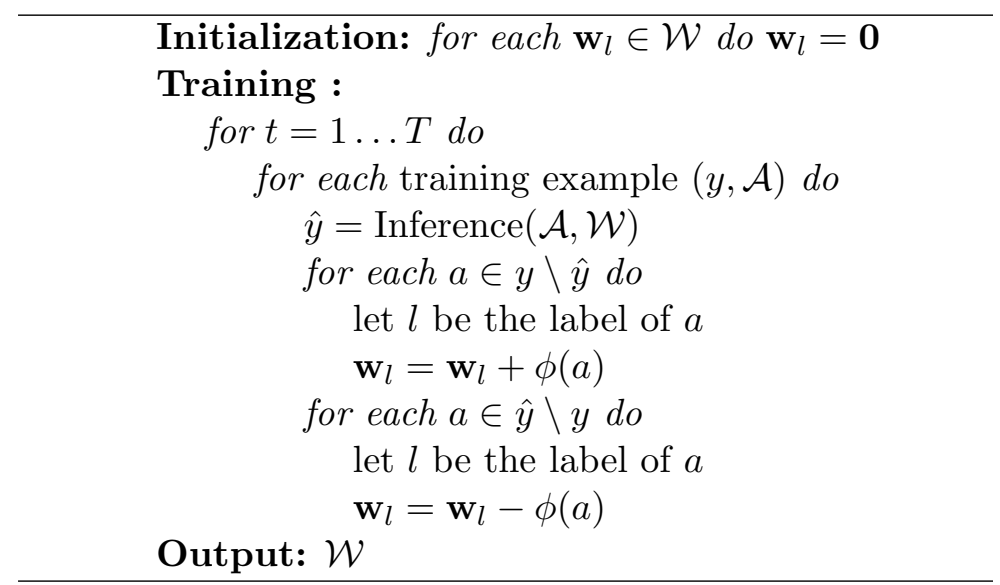

Figure 7: Perceptron Global Learning Algorithm

$\phi(a)$ the vector of features described in Section 6. With each example, Perceptron performs two steps. First, it predicts the optimal solution $\hat{y}$ according to the current setting of $\mathcal{W}$. Note that the prediction strategy employs the complete combination model, including the inference component. Second, Perceptron corrects the vectors in $\mathcal{W}$ according to the mistakes seen in $\hat{y}$ : arguments with label $l$ seen in $y$ and not in $\hat{y}$ are promoted in vector $\mathbf{w}_{l}$; on the other hand, arguments in $\hat{y}$ and not in $y$ are demoted in $\mathbf{w}_{l}$. This correction rule moves the scoring vectors towards missing arguments, and away from predicted arguments that are not correct. It is guaranteed that, as Perceptron visits more and more examples, this feedback rule will improve the accuracy of the global combination function when the feature space is almost linearly separable (Freund \& Schapire, 1999; Collins, 2002).

In all, this training strategy is global because the mistakes that Perceptron corrects are those that arise when comparing the predicted structure with the correct one. In contrast, a local strategy identifies mistakes looking individually at the sign of scoring predictions: if some candidate argument is (is not) in the correct solution and the current scorers predict a negative (positive) confidence value, then the corresponding scorer is corrected with that candidate argument. Note that this is the same criteria used to generate training data for classifiers trained locally. In Section 8 we compare these approaches empirically.

As a final note, for simplicity we have described Perceptron in its most simple form. However, the Perceptron version we use in the experiments reported in Section 8 incorporates two well-known extensions: kernels and averaging (Freund \& Schapire, 1999; Collins \& Duffy, 2002). Similar to SVM, Perceptron is a kernel method. That is, it can be represented in dual form, and the dot product between example vectors can be generalized by a kernel function that exploits richer representations. On the other hand, averaging is a technique that increases the robustness of predictions during testing. In the original form, test predictions are computed with the parameters that result from the training process. In the averaged version, test predictions are computed with an average of all parameter vectors that are generated during training, after every update. Details of the technique can be found in the original article of Freund \& Schapire. 


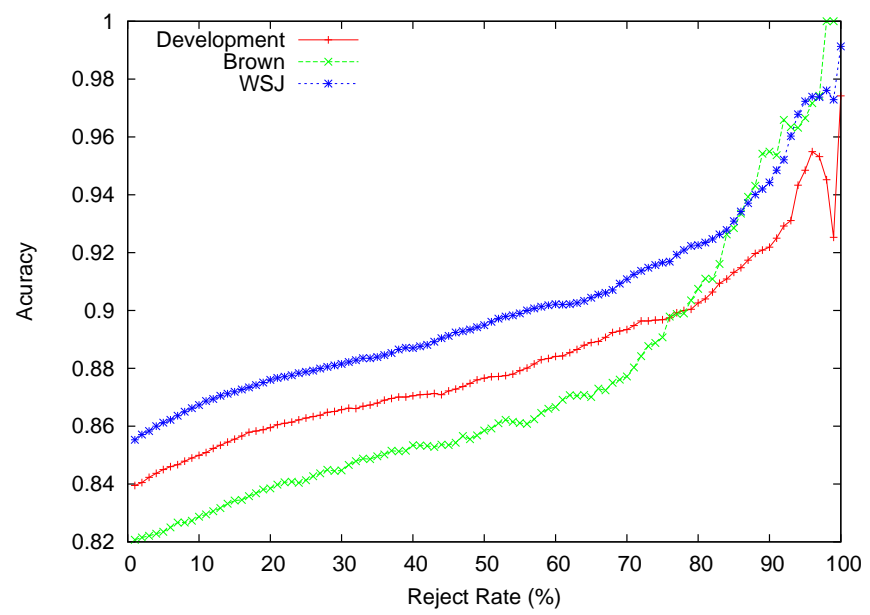

Figure 8: Rejection curves of the estimated output probabilities of the individual models.

\section{Experimental Results}

In this section we analyze the performance of the three combination strategies previously described: (a) inference with constraint satisfaction, (b) learning-based inference with local rankers, and (c) learning-based inference with global rankers. For the bulk of the experiments we use candidate arguments generated by the three individual SRL models described in Section 4 and evaluated in Section 5.

\subsection{Experimental Settings}

All combination strategies (with one exception, detailed below) were trained using the complete CoNLL-2005 training set (PropBank/Treebank sections 2 to 21). To minimize the overfitting of the individual SRL models on the training data, we partitioned the training corpus into five folds and for each fold we used the output of the individual models when trained on the remaining four folds. The models were tuned on a separate development partition (Treebank section 24) and evaluated on two corpora: (a) Treebank section 23, and (b) on the three annotated sections of the Brown corpus.

For the constraint satisfaction model, we converted the scores of the output arguments of the three SRL models into probabilities using the softmax function explained in Section 7.1. The development set (section 24) was used to tune the $\gamma$ parameter of the softmax formula to a final value of 0.1 for all models. In order to assess the quality of this procedure, we plot in Figure 8 the rejection curves of the estimated output probabilities with respect to classification accuracy on the development and test sets (WSJ and Brown). To calculate these plots, the probability estimates of all three models are put together in a set and sorted in decreasing order. At a certain level of rejection $(n \%)$, the curve in Figure 8 plots the percentage of correct arguments when the lowest scoring $n \%$ subset is rejected. With few 
exceptions, the curves are increasing and smooth, indicating a good correlation between probability estimates and classification accuracy.

As a last experiment, in Section 8.6 we analyze the behavior of the proposed combination strategies when the candidate pool is significantly larger. For this experiment we used the top 10 best performing systems at the CoNLL-2005 shared task evaluation. In this setup there are two significant differences from the experiments that used our in-house individual systems: (a) we had access only to the systems' outputs on the PropBank development section and on the two test sections, and (b) the argument probabilities of the individual models were not available. Thus, instead of the usual training set, we had to train our combination models on the PropBank development section with a smaller feature set. Note also that the development set is only $3.45 \%$ of the size of the regular training set. We evaluated the resulting combination models on the same two testing sections: WSJ and Brown.

\subsection{Lower and Upper Bounds of the Combination Strategies}

Before we venture into the evaluation of the combination strategies, we explore the lower and upper bounds of the combinations models on the given corpus and individual models. This analysis is important in order to understand the potential of the proposed approach and to see how close we actually are to realizing it.

The performance upper bound is calculated with an oracle combination system with a perfect filtering classifier that selects only correct candidate arguments and discards all others. For comparison purposes, we have implemented a second oracle system that simulates a re-ranking approach: for each predicate it selects the candidate frame -i.e., the complete set of arguments for the corresponding predicate proposed by a single modelwith the highest $\mathrm{F}_{1}$ score. Table 3 lists the results obtained on the WSJ and Brown corpora by these two oracle systems using all three individual models. The "combination" system is the oracle that simulates the combination strategies proposed in this paper, which break candidate frames and work with individual candidate arguments. Note that the precision of this oracle combination system is not $100 \%$ because in the case of discontinuous arguments, fragments that pass the oracle filter are considered incorrect by the scorer when the corresponding argument is not complete, e.g., an argument A1 appears without the continuation $\mathrm{C}-\mathrm{A} 1$. The "re-ranking" columns list the results of the second oracle system, which selects entire candidate frames.

Table 3 indicates that the upper limit of the combination approaches proposed in this paper is relatively high: the $\mathrm{F}_{1}$ of the combination oracle system is over 14 points higher than our best individual system in the WSJ test set, and over 17 points higher in the Brown corpus (see Table 1). Furthermore, our analysis indicates that the potential of our combination strategy is higher than that of re-ranking strategies, which are limited to the performance of the best complete frame in the candidate pool. By allowing the recombination of arguments from the individual candidate solutions this threshold is raised significantly: over $6 \mathrm{~F}_{1}$ points in WSJ and over $9 \mathrm{~F}_{1}$ points in Brown.

Table 4 lists the distribution of the candidate arguments from the individual models in the selection performed by the combination oracle system. For conciseness, we list only the core numbered arguments and we focus on the WSJ corpus. " $\cap$ of 3 " indicates the percent- 


\begin{tabular}{|l|c|c|c|c||c|c|c|c|}
\cline { 2 - 9 } \multicolumn{1}{c|}{} & \multicolumn{4}{c||}{ Combination } & \multicolumn{4}{c|}{ Re-Ranking } \\
\cline { 2 - 9 } \multicolumn{1}{c|}{} & PProps & Precision & Recall & $\mathrm{F}_{1}$ & PProps & Precision & Recall & $\mathrm{F}_{1}$ \\
\hline WSJ & $70.76 \%$ & $99.12 \%$ & $85.22 \%$ & 91.64 & $63.51 \%$ & $88.08 \%$ & $82.84 \%$ & 85.38 \\
Brown & $51.87 \%$ & $99.63 \%$ & $74.32 \%$ & 85.14 & $45.02 \%$ & $80.80 \%$ & $71.70 \%$ & 75.98 \\
\hline
\end{tabular}

Table 3: Performance upper limits detected by the two oracle systems.

\begin{tabular}{|l|c|c|c|c|c|}
\cline { 2 - 6 } \multicolumn{1}{c|}{} & $\cap$ of 3 & $\cap$ of 2 & Model 1 & Model 2 & Model 3 \\
\hline A0 & $80.45 \%$ & $12.10 \%$ & $3.47 \%$ & $2.14 \%$ & $1.84 \%$ \\
A1 & $69.82 \%$ & $17.83 \%$ & $7.45 \%$ & $2.77 \%$ & $2.13 \%$ \\
A2 & $56.04 \%$ & $22.32 \%$ & $12.20 \%$ & $4.95 \%$ & $4.49 \%$ \\
A3 & $56.03 \%$ & $21.55 \%$ & $12.93 \%$ & $5.17 \%$ & $4.31 \%$ \\
A4 & $65.85 \%$ & $20.73 \%$ & $6.10 \%$ & $2.44 \%$ & $4.88 \%$ \\
\hline
\end{tabular}

Table 4: Distribution of the individual systems' arguments in the upper limit selection, for $\mathrm{A} 0-\mathrm{A} 4$ in the WSJ test set.

age of correct arguments where all 3 models agreed, " $\cap$ of 2 " indicates the percentage of correct arguments where any 2 models agreed, and the other columns indicate the percentage of correct arguments detected by a single model. Table 4 indicates that, as expected, two or more individual models agreed on a large percentage of the correct arguments. Nevertheless, a significant number of correct arguments, e.g., over $22 \%$ of A3, come from a single individual system. This proves that, in order to achieve maximum performance, one has to look beyond simple voting strategies that favor arguments with high agreement between individual systems.

We propose two lower bounds for the performance of the combination models using two baseline systems:

- The first baseline is recall-oriented: it merges all the arguments generated by the individual systems. For conflict resolution, the baseline uses an approximate inference algorithm consisting of two steps: (i) candidate arguments are sorted using a radix sort that orders the candidate arguments in descending order of: (a) number of models that agreed on this argument, (b) argument length in tokens, and (c) performance of the individual system ${ }^{10}$; (ii) Candidates are iteratively appended to the global solution only if they do not violate any of the domain constraints with the arguments already selected.

- The second baseline is precision-oriented: it considers only arguments where all three individual systems agreed. For conflict resolution it uses the same strategy as the previous baseline system.

Table 5 shows the performance of these two baseline models. As expected, the precisionoriented baseline obtains a precision significantly higher than the best individual model (Table 1), but its recall suffers because the individual models do not agree on a fairly large number of candidate arguments. The recall-oriented baseline is more balanced: as expected the recall is higher than any individual model and the precision does not drop too much

10. This combination produced the highest-scoring baseline model. 


\begin{tabular}{|c|c|c|c|c|}
\hline WSJ & PProps & Prec. & Recall & $\mathrm{F}_{1}$ \\
\hline baseline recall & $53.71 \%$ & $78.09 \%$ & $\mathbf{7 8 . 7 7} \%$ & $78.43 \pm 0.8$ \\
\hline baseline precision & $35.43 \%$ & $\mathbf{9 2 . 4 9} \%$ & $60.48 \%$ & $73.14 \pm 0.9$ \\
\hline \multicolumn{5}{|l|}{ Brown } \\
\hline baseline recall & $\mathbf{3 6 . 9 4} \%$ & $68.57 \%$ & $66.05 \%$ & $67.29 \pm 2.0$ \\
\hline baseline precision & $20.52 \%$ & $88.74 \%$ & $46.35 \%$ & $60.89 \pm 2.1$ \\
\hline
\end{tabular}

Table 5: Performance of the baseline models on the WSJ and Brown test sets.

because the inference strategy filters out many unlikely candidates. Overall, the recalloriented baseline performs best, with an $\mathrm{F}_{1} 1.22$ points higher than the best individual model on the WSJ corpus, and 0.41 points lower on the Brown corpus.

\subsection{Performance of the Combination System with Constraint Satisfaction}

In the Constraint Satisfaction setting the arguments output by individual Models 1, 2, and 3 are recombined into an expected better solution that satisfies a set of constraints. We have run the inference model based on Constraint Satisfaction described in Section 7.1 using the Xpress-MP ILP solver ${ }^{11}$. The main results are summarized in Table 6 . The variants presented in that table are the following: "Pred-by-pred" stands for local optimization, which processes each verb predicate independently from others, while "Full sentence" stands for global optimization, i.e., resolving all the verb predicates of the sentence at the same time. The column labeled "Constraints" shows the particular constraints applied at each configuration. The "O" column presents the value of the parameter for correcting the bias towards candidate overgeneration. Concrete values are empirically set to maximize the $\mathrm{F}_{1}$ measure on the development set. $O=0$ corresponds to a setting in which no bias correction is applied.

Some clear conclusions can be drawn from Table 6. First, we observe that any optimization variant obtains $F_{1}$ results above both the individual systems (Table 1 ) and the baseline combination schemes (Table 5 ). The best combination model scores $2.61 \mathrm{~F}_{1}$ points in WSJ and 1.49 in Brown higher than the best individual system. Taking into account that no learning is performed, it is clear that Constraint Satisfaction is a simple yet formal setting that achieves good results.

A somewhat surprising result is that all performance improvements come from constraints 1 and 2 (i.e., no overlapping nor embedding among arguments of the same verb, and no repetition of core arguments in the same verb). Constraints 3 and 4 are harmful, while the sentence-level constraints ( 5 and 6 ) have no impact on the overall performance ${ }^{12}$. Our analysis of the proposed constraints yielded the following explanations:

- Constraint number 3 prevents the assignment of an $\mathrm{R}-\mathrm{X}$ argument when the referred argument $\mathrm{X}$ is not present. This makes the inference miss some easy $\mathrm{R}-\mathrm{X}$ arguments

11. Xpress-MP is a Dash Optimization product that is free for academic usage.

12. In Section 8.5 we will see that when the learning strategy incorporates global feedback, performing a sentence-level inference is slightly better than proceeding predicate by predicate. 


\begin{tabular}{|l|l|l|l|c|c|c|}
\cline { 2 - 6 } \multicolumn{1}{c|}{ WSJ } & Constraints & $O$ & PProps & Precision & Recall & $\mathrm{F}_{1}$ \\
\hline Pred-by-pred & 1 & 0.30 & $52.29 \%$ & $84.20 \%$ & $75.64 \%$ & $79.69 \pm 0.8$ \\
& $1+2$ & 0.30 & $52.52 \%$ & $84.61 \%$ & $75.53 \%$ & $79.81 \pm 0.6$ \\
& $1+2+3$ & 0.25 & $52.31 \%$ & $84.34 \%$ & $75.48 \%$ & $79.67 \pm 0.7$ \\
& $1+2+4$ & 0.30 & $51.40 \%$ & $84.13 \%$ & $75.04 \%$ & $79.32 \pm 0.8$ \\
& $1+2+3+4$ & 0.30 & $51.19 \%$ & $83.86 \%$ & $74.99 \%$ & $79.18 \pm 0.7$ \\
\hline Full sentence & $1+2+5$ & 0.30 & $52.53 \%$ & $84.63 \%$ & $73.53 \%$ & $\mathbf{7 9 . 8 2} \pm 0.7$ \\
& $1+2+6$ & 0.30 & $52.48 \%$ & $84.64 \%$ & $75.51 \%$ & $79.81 \pm 0.8$ \\
& $1+2+5+6$ & 0.30 & $52.50 \%$ & $\mathbf{8 4 . 6 5 \%}$ & $75.51 \%$ & $\mathbf{7 9 . 8 2} \pm 0.6$ \\
& $1+2+5+6$ & 0 & $\mathbf{5 4 . 4 9} \%$ & $78.74 \%$ & $\mathbf{7 9 . 7 8} \%$ & $79.26 \pm 0.7$ \\
\hline Brown & $1+2+5+6$ & 0.30 & $35.70 \%$ & $\mathbf{7 8 . 1 8} \%$ & $62.06 \%$ & $\mathbf{6 9 . 1 9} \pm 2.1$ \\
\hline Full sentence & $1+2+5+6$ & 0 & $\mathbf{3 8 . 0 6 \%}$ & $69.80 \%$ & $\mathbf{6 7 . 8 5} \%$ & $68.81 \pm 2.2$ \\
\hline
\end{tabular}

Table 6: Results, on WSJ and Brown test sets, obtained by multiple variants of the constraint satisfaction approach

when the X argument is not correctly identified (e.g., constituents that start with $\{$ that, which, who\} followed by a verb are always R-A0). Furthermore, this constraint presents a lot of exceptions: up to $18.75 \%$ of the $\mathrm{R}-\mathrm{X}$ arguments in the WSJ test set do not have the referred argument X (e.g., "when" in "the law tells them when to do so"), therefore the hard application of constraint 3 prevents the selection of some correct $\mathrm{R}-\mathrm{X}$ candidates. The official evaluation script from CoNLL-2005 (srl-eval) does not require this constraint to be satisfied to consider a solution consistent.

- The srl-eval script requires that constraint number 4 (i.e., a C-X tag is not accepted without a preceding $\mathrm{X}$ argument) be fulfilled for a candidate solution to be considered consistent. But when it finds a solution violating the constraint its behavior is to convert the first $\mathrm{C}-\mathrm{X}$ (without a preceding $\mathrm{X}$ ) into $\mathrm{X}$. It turns out that this simple post-processing strategy is better than forcing a coherent solution in the inference step because it allows to recover from the error when an argument has been completely recognized but labeled only with $\mathrm{C}-\mathrm{X}$ tags.

- Regarding sentence-level constraints, we observed that in our setting, inference using local constraints $(1+2)$ rarely produces a solution with inconsistencies at sentence level. ${ }^{13}$ This makes constraint 5 useless since it is almost never violated. Constraint number 6 (i.e., no sharing of AMs among different verbs) is more ad-hoc and represents a less universal principle in SRL. The number of exceptions to that constraint, in the WSJ test set, is $3.0 \%$ for the gold-standard data and $4.8 \%$ in the output of the inference that uses only local constraints $(1+2)$. Forcing the fulfillment of this constraint makes the inference process commit as many errors as corrections, making its effect negligible.

13. This fact is partly explained by the small number of overlapping arguments in the candidate pool produced by the three individual models. 

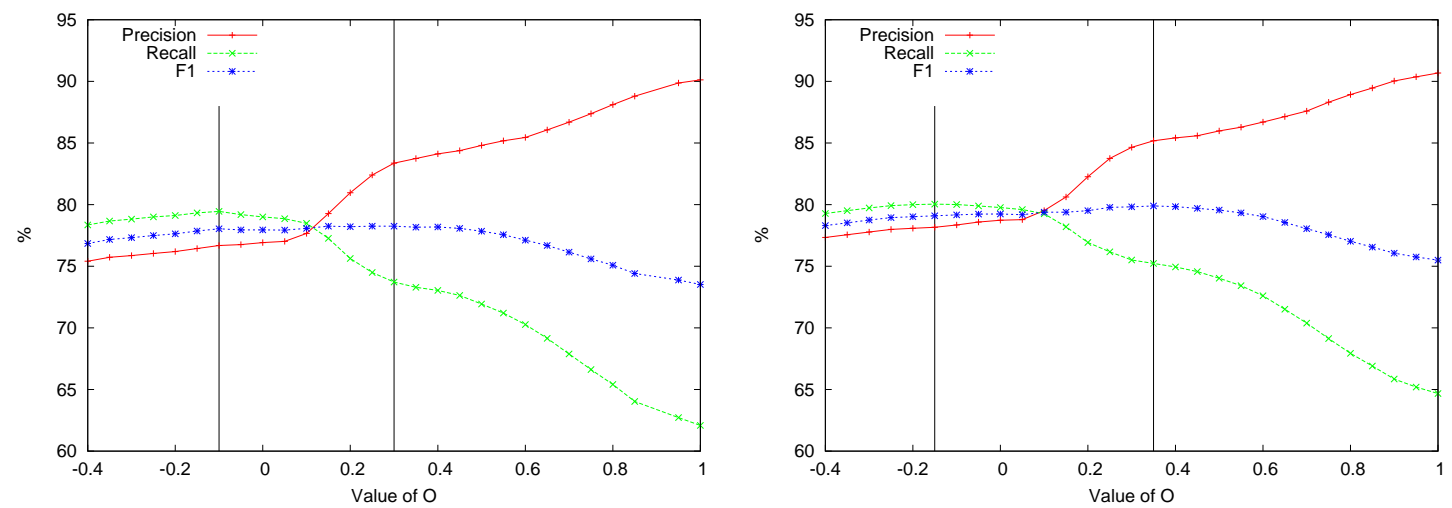

Figure 9: Precision-Recall plots, with respect to the bias correcting parameter $(O)$, for the WSJ development and test sets (left and right plots, respectively).

Considering that some of the constraints are not universal, i.e., exceptions exist in the gold standard, it seems reasonable to convert them into soft constraints. This can be done by precomputing their compatibility from corpora counts using, for instance, point-wise mutual information, and incorporating its effect in the compatibility function as explained in section 7.1. This softening could, in principle, increase the overall recall of the combination. Unfortunately, our initial experiments showed no differences between the hard and soft variants.

Finally, the differences between the optimized values of the bias correcting parameter and $O=0$ are clearly explained by observing precision and recall values. The default version tends to overgenerate argument assignments, which implies a higher recall at a cost of a lower precision. On the contrary, the $\mathrm{F}_{1}$ optimized variant is more conservative and needs more evidence to select a candidate. As a result, the precision is higher but the recall is lower. A side effect of being restrictive with argument assignments, is that the number of correctly annotated complete propositions is also lower in the optimized setting.

The preference for a high-precision vs. a high-recall system is mostly task-dependant. It is interesting to note that in this constraint satisfaction setting, adjusting the precisionrecall tradeoff can be easily done by varying the value of the bias correcting score. In Figure 9, we plot the precision-recall curves with respect to different values of the $O$ parameter (the optimization is done using constraints 1, 2, 5, and 6). As expected, high values of $O$ promote precision and demote recall, while lower values of $O$ do just the contrary. Also, we see that there is a wide range of values for which the combined $F_{1}$ measure is almost constant (the approximate intervals are marked using vertical lines), making it possible to select different recall and precision values with a global performance $\left(F_{1}\right)$ near to the optimal. Parenthetically, note also that the optimal value estimated on the development set $(O=0.3)$ generalizes very well to the WSJ test set. 


\begin{tabular}{|c|c|c|c|c|c|}
\hline WSJ & PProps & Prec. & Recall & $\mathrm{F}_{1}$ & $\mathrm{~F}_{1}$ improvement \\
\hline Models $1+2$ & $49.28 \%$ & $87.39 \%$ & $72.88 \%$ & $79.48 \pm 0.6$ & +2.27 \\
\hline Models $1+3$ & $48.26 \%$ & $86.80 \%$ & $73.20 \%$ & $79.42 \pm 0.6$ & +2.96 \\
\hline Models $2+3$ & $49.36 \%$ & $86.63 \%$ & $73.03 \%$ & $79.25 \pm 0.7$ & +2.04 \\
\hline Models $1+2+3$ & $51.66 \%$ & $87.47 \%$ & $74.67 \%$ & $\mathbf{8 0 . 5 6} \pm 0.6$ & +3.35 \\
\hline \multicolumn{6}{|l|}{ Brown } \\
\hline Models $1+2$ & $34.33 \%$ & $81.14 \%$ & $60.86 \%$ & $69.55 \pm 2.0$ & +1.85 \\
\hline Models $1+3$ & $31.22 \%$ & $80.43 \%$ & $59.07 \%$ & $68.11 \pm 1.9$ & +2.69 \\
\hline Models $2+3$ & $32.84 \%$ & $80.90 \%$ & $60.31 \%$ & $69.11 \pm 2.1$ & +1.41 \\
\hline Models $1+2+3$ & $34.33 \%$ & $81.75 \%$ & $61.32 \%$ & $\mathbf{7 0 . 0 8} \pm 2.1$ & +2.38 \\
\hline
\end{tabular}

Table 7: Overall results of the learning-based inference with local rankers on the WSJ and Brown test sets.

\subsection{Performance of the Combination System with Local Rankers}

We implemented the candidate-scoring classifiers for this combination strategy using Support Vector Machines (SVM) with polynomial kernels of degree 2, which performed slightly better than other types of SVMs or AdaBoost. We have implemented the SVM classifiers with the SVM ${ }^{\text {light }}$ software ${ }^{14}$. Outside of changing the default kernel to polynomial we have not modified the default parameters. For the experiments reported in this section, we trained models for all 4 possible combinations of our 3 individual systems, using the complete feature set introduced in Section 6. The dynamic programming engine used for the actual inference processes each predicate independently (similar to the "Pred-by-pred" approach in the previous sub-section).

Table 7 summarizes the performance of the combined systems on the WSJ and Brown corpora. Table 7 indicates that our combination strategy is always successful: the results of all combination systems improve upon their individual models (Table 1) and their $\mathrm{F}_{1}$ scores are always better than the baselines' (Table 5). The last column in the table shows the $\mathrm{F}_{1}$ improvement of the combination model w.r.t. the best individual model in each set. As expected, the highest scoring combined system includes all three individual models. Its $\mathrm{F}_{1}$ measure is 3.35 points higher than the best individual model (Model 2) in the WSJ test set and 2.38 points higher in the Brown test set. Note that with any combination of two individual systems we outperform the current state of the art (see Section 9 for details). This is empirical proof that robust and successful combination strategies for the SRL problem are possible. Table 7 also indicates that, even though the partial parsing model (Model 1) is the worst performing individual model, its contribution to the ensemble is very important, indicating that the information it provides is indeed complementary to the other models'. For instance, in WSJ the performance of the combination of the two best individual models (Models 2+3) is worse than the combinations using model 1 (Models $1+2$ and $1+3$ ).

14. http://svmlight.joachims.org/ 


\begin{tabular}{|c|c|c|c|c|}
\hline WSJ & PProps & Prec. & Recall & $\mathrm{F}_{1}$ \\
\hline FS1 & $50.24 \%$ & $86.47 \%$ & $73.51 \%$ & $79.47 \pm 0.7$ \\
\hline$+\mathrm{FS} 2$ & $50.39 \%$ & $86.41 \%$ & $73.68 \%$ & $79.54 \pm 0.6$ \\
\hline+ FS3 & $51.22 \%$ & $86.13 \%$ & $74.35 \%$ & $79.80 \pm 0.7$ \\
\hline$+\mathrm{FS} 4$ & $50.66 \%$ & $86.67 \%$ & $74.10 \%$ & $79.89 \pm 0.7$ \\
\hline+ FS5 & $51.38 \%$ & $87.21 \%$ & $74.61 \%$ & $80.42 \pm 0.6$ \\
\hline+ FS6 & $51.66 \%$ & $87.47 \%$ & $74.67 \%$ & $\mathbf{8 0 . 5 6} \pm 0.6$ \\
\hline \multicolumn{5}{|l|}{ Brown } \\
\hline FS1 & $32.21 \%$ & $80.12 \%$ & $59.44 \%$ & $68.25 \pm 2.0$ \\
\hline$+\mathrm{FS} 2$ & $32.84 \%$ & $80.80 \%$ & $59.94 \%$ & $68.83 \pm 2.2$ \\
\hline+ FS3 & $33.33 \%$ & $80.29 \%$ & $60.82 \%$ & $69.21 \pm 2.0$ \\
\hline$+\mathrm{FS} 4$ & $33.33 \%$ & $81.10 \%$ & $60.50 \%$ & $69.30 \pm 2.1$ \\
\hline+ FS5 & $34.08 \%$ & $81.76 \%$ & $61.14 \%$ & $69.96 \pm 2.2$ \\
\hline$+\mathrm{FS} 6$ & $34.33 \%$ & $81.75 \%$ & $61.32 \%$ & $\mathbf{7 0 . 0 8} \pm 1.9$ \\
\hline
\end{tabular}

Table 8: Feature analysis for the learning-based inference with local rankers.

Due to its simple architecture -i.e., no feedback from the conflict resolution component to candidate filtering - this inference model is a good framework to study the contribution of the features proposed in Section 6. For this study we group the features into 6 sets: FS1 - the voting features, FS2 - the overlap features with arguments for the same predicate, FS3 - the overlap features with arguments for other predicates, FS4 - the partial-syntax features, FS5 -the full-syntax features, and FS6 - the probabilities generated by the individual systems for the candidate arguments. Using these sets we constructed 6 combination models by increasing the number of features made available to the argument filtering classifiers, e.g., the first system uses only FS1, the second system adds FS2 to the first system's features, FS3 is added for the third system, etc. Table 8 lists the performance of these 6 systems for the two test corpora. This empirical analysis indicates that the feature sets with the highest contribution are:

- FS1, which boosts the $\mathrm{F}_{1}$ score of the combined system 2.26 points (WSJ) and 0.55 points (Brown) over our best individual system. This is yet another empirical proof that voting is a successful combination strategy.

- FS5, with a contribution of 0.53 points (WSJ) and 0.66 points (Brown) to the $\mathrm{F}_{1}$ score. These numbers indicate that the filtering classifier is capable of learning some of the "preferences" of the individual models for certain syntactic structures.

- FS3, which contributes 0.26 points (WSJ) and 0.38 points (Brown) to the $\mathrm{F}_{1}$ score. These results promote the idea that information about the overall sentence structure, in our case inter-predicate relations, can be successfully used for the problem of SRL. To our knowledge, this is novel.

All the proposed features have a positive contribution to the performance of the combined system. Overall, we achieve an $\mathrm{F}_{1}$ score that is 1.12 points (WSJ) and 2.33 points (Brown) higher than the best performing combined system at the CoNLL-2005 shared task evaluation (see Section 9 for details). 


\subsection{Performance of the Combination System with Global Rankers}

In this section we report experiments with the global Perceptron algorithm described in Section 7.2.2, that globally trains the scoring functions as rankers. Similar to the local SVM models, we use polynomial kernels of degree 2. Furthermore, the predictions at test time used averages of the parameter vectors, following the technique of Freund and Schapire (1999).

We were interested in two main aspects. First, we evaluate the effect of training the scoring functions with Perceptron using two different update rules, one global and the other local. The global feedback rule, detailed in Section 7.2.2, corrects the mistakes found when comparing the correct argument structure with the one that results from the inference (this is noted as "global" feedback). In contrast, the local feedback rule corrects the mistakes found before inference, when each candidate argument is handled independently, ignoring the global argument structure generated (this is noted as "local" feedback). Second, we analyze the effect of using different constraints in the inference module. To this extent, we configured the inference module in two ways. The first processes the predicates of a sentence independently, and thus might select overlapping arguments of different predicates, which is incorrect according to the domain constraints (this one is noted as "Pred-by-pred" inference). The second processes all predicates jointly, and enforces a hierarchical structure of arguments, where arguments never overlap, and arguments of a predicate are allowed to embed arguments of other predicates (this is noted as "Full sentence" inference). From this perspective, the model with local update and "Pred-by-pred" inference is almost identical to the local combination strategy described in Section 8.4, with the unique difference that here we use Perceptron instead of SVM. This apparently minute difference turns out to be significant for our empirical analysis because it allows us to measure the contribution of both SVM margin maximization and global feedback to the classifier-based combination strategy (see Section 8.7).

We trained four different models: with local or global feedback, and with predicate-bypredicate or joint inference. Each model was trained for 5 epochs on the training data, and evaluated on the development data after each training epoch. We selected the best performing point on development, and evaluated the models on the test data. Table 9 reports the results on test data.

Looking at results, a first impression is that the difference in $F_{1}$ measure is not very significant among different configurations. However, some observations can be pointed out. Global methods achieve much better recall figures, whereas local methods prioritize the precision of the system. Overall, global methods achieve a more balanced tradeoff between precision and recall, which contributes to a better $\mathrm{F}_{1}$ measure.

Looking at "Pred-by-pred" versus "Full sentence" inference, it can be seen that only the global methods are sensitive to the difference. Note that a local model is trained independently of the inference module. Thus, adding more constraints to the inference engine does not change the parameters of the local model. At testing time, the different inference configurations do not affect the results. In contrast, the global models are trained dependently of the inference module. When moving from "Pred-by-pred" to "Full sentence" inference, consistency is enforced between argument structures of different predicates, and this benefits both the precision and recall of the method. The global learning algorithm 


\begin{tabular}{|c|c|c|c|c|}
\hline WSJ & PProps & Prec. & Recall & $\mathrm{F}_{1}$ \\
\hline Pred-by-pred, local & $50.71 \%$ & $86.80 \%$ & $74.31 \%$ & $80.07 \pm 0.7$ \\
\hline Full sentence, local & $50.67 \%$ & $86.80 \%$ & $74.29 \%$ & $80.06 \pm 0.7$ \\
\hline Pred-by-pred, global & $53.45 \%$ & $84.66 \%$ & $76.19 \%$ & $80.20 \pm 0.7$ \\
\hline Full sentence, global & $53.81 \%$ & $84.84 \%$ & $76.30 \%$ & $\mathbf{8 0 . 3 4} \pm 0.6$ \\
\hline \multicolumn{5}{|l|}{ Brown } \\
\hline Pred-by-pred, local & $33.33 \%$ & $80.62 \%$ & $60.77 \%$ & $69.30 \pm 1.9$ \\
\hline Full sentence, local & $33.33 \%$ & $80.67 \%$ & $60.77 \%$ & $69.32 \pm 2.0$ \\
\hline Pred-by-pred, global & $35.20 \%$ & $77.65 \%$ & $62.70 \%$ & $69.38 \pm 1.9$ \\
\hline Full sentence, global & $35.95 \%$ & $77.91 \%$ & $63.02 \%$ & $\mathbf{6 9 . 6 8} \pm 2.0$ \\
\hline
\end{tabular}

Table 9: Test results of the combination system with global rankers. Four configurations are evaluated, that combine "Pred-by-pred" or "Full sentence" inference with "local" or "global" feedback.

improves both in precision and recall when coupled with a joint inference process that considers more constraints in the solution.

Nevertheless, the combination system with local SVM classifiers, presented in the previous section, achieves marginally better $\mathrm{F}_{1}$ score than the global learning method $(80.56 \%$ vs. $80.34 \%$ in WSJ). This is explained by the different machine learning algorithms (we discuss this issue in detail in Section 8.7). The better $F_{1}$ score is accomplished by a much better precision in the local approach $(87.47 \%$ vs. $84.84 \%$ in WSJ), whereas the recall is lower in the local than in the global approach $(74.67 \%$ vs. $76.30 \%$ in WSJ). On the other hand, the global strategy produces more completely-correct annotations (see the "PProps" column) than any of the local strategies investigated (see Tables 9 and 7). This is to be expected, considering that the global strategy optimizes a sentence-level cost function. Somewhat surprisingly, the number of perfect propositions generated by the global strategy is lower than the number of perfect propositions produced by the constraint-satisfaction approach. We discuss this result in Section 8.7.

\subsection{Scalability of the Combination Strategies}

All the combination experiments reported up to this point used the candidate arguments generated by the three individual SRL models introduced in Section 4 . While these experiments do provide an empirical comparison of the three inference models proposed, they do not answer an obvious scalability question: how do the proposed combination approaches scale when the number of candidate arguments increases but their quality diminishes? We are mainly interested in answering this question for the last two combination models (which use inference based on learning with local or global rankers) for two reasons: (a) they performed better than the constraint satisfaction model in our previous experiments, and (b) because they have no requirements on the individual SRL systems' outputs -unlike the constraint satisfaction model which requires the argument probabilities of the individual models - they can be coupled to pools of candidates generated by any individual SRL model. 


\begin{tabular}{|c|c|c|c|c|c|c|c|c|c|}
\hline & \multicolumn{4}{|c|}{ WSJ } & \multicolumn{4}{|c|}{ Brown } \\
\hline & & PProps & Prec. & Recall & $\mathrm{F}_{1}$ & PProps & Prec. & Recall & $\mathrm{F}_{1}$ \\
\hline koomen & $\sqrt{ }$ & $53.79 \%$ & $82.28 \%$ & $76.78 \%$ & 79.44 & $32.34 \%$ & $73.38 \%$ & $62.93 \%$ & 67.75 \\
\hline pradhan+ & $\sqrt{ }$ & $52.61 \%$ & $82.95 \%$ & $74.75 \%$ & 78.63 & $\mathbf{3 8 . 9 3} \%$ & $\mathbf{7 4 . 4 9} \%$ & $63.30 \%$ & 68.44 \\
\hline haghighi & $\sqrt{ }$ & $\mathbf{5 6 . 5 2} \%$ & $79.54 \%$ & $\mathbf{7 7 . 3 9} \%$ & 78.45 & $37.06 \%$ & $70.24 \%$ & $\mathbf{6 5 . 3 7 \%}$ & 67.71 \\
\hline marquez & $\sqrt{ }$ & $51.85 \%$ & $79.55 \%$ & $76.45 \%$ & 77.97 & $36.44 \%$ & $70.79 \%$ & $64.35 \%$ & 67.42 \\
\hline pradhan & $\times$ & $50.14 \%$ & $81.97 \%$ & $73.27 \%$ & 77.37 & $36.44 \%$ & $73.73 \%$ & $61.51 \%$ & 67.07 \\
\hline surdeanu & $\sqrt{ }$ & $45.28 \%$ & $80.32 \%$ & $72.95 \%$ & 76.46 & $29.48 \%$ & $72.41 \%$ & $59.67 \%$ & 65.42 \\
\hline tsai & $\sqrt{ }$ & $45.43 \%$ & $82.77 \%$ & $70.90 \%$ & 76.38 & $30.47 \%$ & $73.21 \%$ & $59.49 \%$ & 65.64 \\
\hline che & $\sqrt{ }$ & $47.81 \%$ & $80.48 \%$ & $72.79 \%$ & 76.44 & $31.84 \%$ & $71.13 \%$ & $59.99 \%$ & 65.09 \\
\hline moschitti & $\sqrt{ }$ & $47.66 \%$ & $76.55 \%$ & $75.24 \%$ & 75.89 & $30.85 \%$ & $65.92 \%$ & $61.83 \%$ & 63.81 \\
\hline tjongkimsang & $\sqrt{ }$ & $45.85 \%$ & $79.03 \%$ & $72.03 \%$ & 75.37 & $28.36 \%$ & $70.45 \%$ & $60.13 \%$ & 64.88 \\
\hline yi & $x$ & $47.50 \%$ & $77.51 \%$ & $72.97 \%$ & 75.17 & $31.09 \%$ & $67.88 \%$ & $59.03 \%$ & 63.14 \\
\hline ozgencil & $\sqrt{ }$ & $46.19 \%$ & $74.66 \%$ & $74.21 \%$ & 74.44 & $31.47 \%$ & $65.52 \%$ & $62.93 \%$ & 64.20 \\
\hline
\end{tabular}

Table 10: Performance of the best systems at CoNLL-2005. The "pradhan+" contains postevaluation improvements. The top 5 systems are actually combination models themselves. The second column marks with $\times$ the systems not used in our evaluation: "pradhan", which was replaced by its improved version "pradhan+", and "yi", due to format errors in the submitted data.

For this scalability analysis, we use as individual SRL models the top 10 systems at the CoNLL-2005 shared task evaluation. Table 10 summarizes the performance of these systems on the same two test corpora used in our previous experiments. As Table 10 indicates, the performance of these systems varies widely: there is a difference of $5 \mathrm{~F}_{1}$ points in the WSJ corpus and of over $4 \mathrm{~F}_{1}$ points in the Brown corpus between the best and the worst system in this set.

For the combination experiments we generated 5 candidate pools using the top 2, 4, 6 , 8 , and 10 individual systems labeled with $\sqrt{ }$ in Table 10 . We had to make two changes to the experimental setup used in the first part of this section: (a) we trained our combined models on the PropBank development section because we did not have access to the individual systems' outputs on the PropBank training partition; and (b) from the feature set introduced in Section 6 we did not use the individual systems' argument probabilities because the raw activations of the individual models' classifiers were not available. Note that under these settings the size of the training corpus is 10 times smaller than the size of the training set used in the previous experiments.

Table 11 shows the upper limits of these setups using the "combination" and "reranking" oracle systems introduced in Section 8.2. Besides performance numbers, we also list in Table 11 the average number of candidates per sentence for each setup, i.e., number of unique candidate arguments ("\# Args./Sent.") for the "combination" oracle and number of unique candidate frames ("\# Frames/Sent.") for the "re-ranking" oracle. Table 12 lists the performance of our combined models with both local feedback (Section 7.2.1) and global feedback (Section 7.2.2). The combination strategy with global rankers uses joint inference and global feedback (see the description in the previous sub-section). 


\begin{tabular}{|c|c|c|c|c|c|c|c|c|}
\hline \multirow[b]{2}{*}{ WSJ } & \multicolumn{4}{|c|}{ Combination } & \multicolumn{4}{|c|}{ Re-Ranking } \\
\hline & \# Args./Sent. & Prec. & Recall & $\mathrm{F}_{1}$ & \# Frames/Sent. & Prec. & Recall & $\mathrm{F}_{1}$ \\
\hline $\mathrm{C} 2$ & 8.53 & $99.34 \%$ & $82.71 \%$ & 90.27 & 3.16 & $88.63 \%$ & $81.77 \%$ & 85.07 \\
\hline $\mathrm{C} 4$ & 9.78 & $99.47 \%$ & $87.26 \%$ & 92.96 & 4.44 & $91.08 \%$ & $86.12 \%$ & 88.53 \\
\hline $\mathrm{C} 6$ & 10.23 & $99.47 \%$ & $88.02 \%$ & 93.39 & 7.21 & $92.14 \%$ & $86.57 \%$ & 89.27 \\
\hline $\mathrm{C} 8$ & 10.74 & $99.48 \%$ & $88.63 \%$ & 93.75 & 8.11 & $92.88 \%$ & $87.33 \%$ & 90.02 \\
\hline $\mathrm{C} 10$ & 11.33 & $\mathbf{9 9 . 5 0} \%$ & $\mathbf{8 9 . 0 2} \%$ & 93.97 & 8.97 & $93.31 \%$ & $87.71 \%$ & 90.42 \\
\hline \multicolumn{9}{|l|}{ Brown } \\
\hline $\mathrm{C} 2$ & 7.42 & $99.62 \%$ & $71.34 \%$ & 83.14 & 3.02 & $82.45 \%$ & $70.37 \%$ & 75.94 \\
\hline $\mathrm{C} 4$ & 8.99 & $99.65 \%$ & $77.58 \%$ & 87.24 & 4.55 & $86.01 \%$ & $75.98 \%$ & 80.68 \\
\hline C6 & 9.62 & $99.65 \%$ & $79.38 \%$ & 88.37 & 7.09 & $88.19 \%$ & $76.80 \%$ & 82.10 \\
\hline $\mathrm{C} 8$ & 10.24 & $99.66 \%$ & $80.52 \%$ & 89.08 & 8.19 & $88.95 \%$ & $78.04 \%$ & 83.14 \\
\hline $\mathrm{C} 10$ & 10.86 & $99.66 \%$ & $81.72 \%$ & 89.80 & 9.21 & $89.65 \%$ & $79.19 \%$ & 84.10 \\
\hline
\end{tabular}

Table 11: Performance upper limits determined by the oracle systems on the 10 best systems at CoNLL-2005. C $k$ stands for combination of the top $k$ systems from Table 10. "\# Args./Sent." indicates the average number of candidate arguments per sentence for the combination oracle; "\# Frames/Sent." indicates the average number of candidate frames per sentence for the re-ranking oracle. The latter can be larger than the number of systems in the combination because on average there are multiple predicates per sentence.

\begin{tabular}{|c|c|c|c|c|c|c|c|c|}
\hline \multirow[b]{2}{*}{ WSJ } & \multicolumn{4}{|c|}{ Local ranker } & \multicolumn{4}{|c|}{ Global ranker } \\
\hline & PProps & Prec. & Recall & $\mathrm{F}_{1}$ & PProps & Prec. & Recall & $\mathrm{F}_{1}$ \\
\hline $\mathrm{C} 2$ & $50.69 \%$ & $86.60 \%$ & $73.90 \%$ & $79.75 \pm 0.7$ & 52.74 & $84.07 \%$ & $75.38 \%$ & $79.49 \pm 0.7$ \\
\hline $\mathrm{C} 4$ & $55.14 \%$ & $86.67 \%$ & $76.63 \%$ & $81.38 \pm 0.7$ & 54.95 & $84.00 \%$ & $77.19 \%$ & $80.45 \pm 0.7$ \\
\hline C6 & $54.85 \%$ & $87.45 \%$ & $76.34 \%$ & $\mathbf{8 1 . 5 2} \pm 0.6$ & 55.21 & $84.24 \%$ & $77.41 \%$ & $80.68 \pm 0.7$ \\
\hline $\mathrm{C} 8$ & $54.36 \%$ & $87.49 \%$ & $76.12 \%$ & $81.41 \pm 0.6$ & 55.00 & $84.42 \%$ & $77.10 \%$ & $80.59 \pm 0.7$ \\
\hline $\mathrm{C} 10$ & $53.90 \%$ & $87.48 \%$ & $75.81 \%$ & $81.23 \pm 0.6$ & 54.76 & $84.02 \%$ & $\mathbf{7 7 . 4 4} \%$ & $80.60 \pm 0.7$ \\
\hline \multicolumn{9}{|l|}{ Brown } \\
\hline $\mathrm{C} 2$ & $32.71 \%$ & $79.56 \%$ & $60.45 \%$ & $68.70 \pm 1.8$ & 35.32 & $74.88 \%$ & $62.43 \%$ & $68.09 \pm 2.0$ \\
\hline $\mathrm{C} 4$ & $35.95 \%$ & $80.27 \%$ & $63.16 \%$ & $70.69 \pm 2.0$ & 39.30 & $75.63 \%$ & $64.45 \%$ & $69.59 \pm 2.2$ \\
\hline C6 & $35.32 \%$ & $80.94 \%$ & $62.24 \%$ & $70.37 \pm 1.8$ & 37.44 & $76.12 \%$ & $64.58 \%$ & $69.88 \pm 2.0$ \\
\hline $\mathrm{C} 8$ & $35.95 \%$ & $81.98 \%$ & $61.87 \%$ & $70.52 \pm 2.2$ & 38.43 & $76.40 \%$ & $64.08 \%$ & $69.70 \pm 2.2$ \\
\hline $\mathrm{C} 10$ & $36.32 \%$ & $\mathbf{8 2 . 6 1} \%$ & $61.97 \%$ & $\mathbf{7 0 . 8 1} \pm 2.0$ & 37.44 & $75.94 \%$ & $\mathbf{6 4 . 6 8} \%$ & $69.86 \pm 2.0$ \\
\hline
\end{tabular}

Table 12: Local versus global ranking for combinations of the 10 best systems at CoNLL2005. C $k$ stands for combination of the top $k$ systems from Table 10.

We can draw several conclusions from these experiments. First, the performance upper limit of re-ranking is always lower than that that of the argument-based combination strategy, even when the number of candidates is large. For example, when all 10 individual models are used, the $\mathrm{F}_{1}$ upper limit of our approach in the Brown corpus is 89.80 whereas the $\mathrm{F}_{1}$ upper limit for re-ranking is 84.10 . However, the enhanced potential of our combination approach does not imply a significant increase in computational cost: Table 11 shows 
that the number of candidate arguments that must be handled by combination approaches is not that much higher than the number of candidate frames input to the re-ranking system, especially when the number of individual models is high. For example, when all 10 individual models are used, the combination approaches must process around 11 arguments per sentence, whereas re-ranking approaches must handle approximately 9 frames per sentence. The intuition behind this relatively small difference in computational cost is that, even though the number of arguments is significantly larger than the number of frames, the difference between the number of unique candidates for the two approaches is not high because the probability of repeated arguments is higher than the probability of repeated frames.

The second conclusion is that all our combination models boost the performance of the corresponding individual systems. For example, the best 4-system combination achieves an $\mathrm{F}_{1}$ score approximately 2 points higher than the best individual model in both the WSJ and Brown corpus. As expected, the combination models reach a performance plateau around 4-6 individual systems, when the quality of the individual models starts to drop significantly. Nevertheless, considering that the top 4 individual systems use combination strategies themselves and the amount of training data for this experiment was quite small, these results show the good potential of the combination models analyzed in this paper.

The third observation is that the relation previously observed between local and global rankers holds: our combination model with local rankers has better precision, but the model with global rankers always has better recall and generally better PProps score. Overall, the model with local rankers obtains better $F_{1}$ scores and scales better as the number of individual systems increases. We discuss these differences in more detail in the next sub-section.

Finally, Table 11 indicates that the potential recall of this experiment (shown in the left-most block in the table) is higher than the potential recall when combining our three individual SRL systems (see Table 3): $3.8 \%$ higher in the WSJ test set, and $7.4 \%$ higher in the Brown test set. This was expected, considering that both the number and the quality of the candidate arguments in this last experiment is higher. However, even after this improvement, the potential recall of our combination strategies is far from $100 \%$. Thus, combining the solutions of the $N$ best state-of-the-art SRL systems still does not have the potential to properly solve the SRL problem. Future work should focus on recallboosting strategies, e.g., using candidate arguments of the individual systems before the individual complete solutions are generated, because in this step many candidate arguments are eliminated.

\subsection{Discussion}

The experimental results presented in this section indicate that all proposed combination strategies are successful: all three combination models provide statistically significant improvements over the individual models and the baselines in all setups. An immediate (but somewhat shallow) comparison of the three combination strategies investigated indicates that: (a) the best combination strategy for the SRL problem is a max-margin local metalearner; (b) the global ranking approach for the meta-learner is important but it does not 
have the same contribution as a max-margin strategy; and (c) the constraint-satisfaction model performs the worst of all the strategies tried.

However, in most experiments the differences between the combination approaches investigated are small. A more reasonable observation is that each combination strategy has its own advantages and disadvantages and different approaches are suitable for different applications and data. We discuss these differences below.

If the argument probabilities of individual systems are available, the combination model based on constraint satisfaction is an attractive choice: it is a simple, unsupervised strategy that obtains competitive performance. Furthermore, the constraint satisfaction model provides an elegant and customizable framework to tune the balance between precision and recall (see Section 8.3). With this framework we currently obtain the highest recall of all combination models: $3.48 \%$ higher than the best recall obtained by the meta-learning approaches on the WSJ corpus, and $4.83 \%$ higher than the meta-learning models on the Brown corpus. The higher recall implies also higher percentage of predicates that are completely correctly annotated: the best "PProps" numbers in Table 6 are the best of all combination strategies. The cause for this high difference in recall in favor of the constraint satisfaction approach is that the candidate scoring of the learning-based inference acts implicitly as a filter: all candidates whose score -i.e., the classifier confidence that the candidate is part of the correct solution - is negative are discarded, which negatively affects the overall recall. Hence, constraint satisfaction is a better solution for SRL-based NLP applications which require that predicate-argument frames be extracted with high recall. For example, in Information Extraction, predicate-argument tuples are filtered with subsequent high-precision, domain-specific constraints (Surdeanu et al., 2003), hence it is paramount that the SRL model have high recall.

Nevertheless, in many cases the argument probabilities of the individual SRL models are not available, either because the models do not generate them, e.g., rule-based systems, or because the individual models are available only as black boxes, which do not offer access to internal information. Under these conditions, we showed that combination strategies based on meta-learning are a viable alternative. In fact, these approaches obtain the highest $\mathrm{F}_{1}$ scores (see Section 8.4) and obtain excellent performance even with small amounts of training data (see Section 8.6). As previously mentioned, because candidate scoring acts as filter, the learning-based inference tends to favor precision over recall: their precision is $2.82 \%$ higher than the best precision of the constraint-satisfaction models in the WSJ corpus, and $3.57 \%$ higher in the Brown corpus. This preference for precision over recall is more pronounced in the learning-based inference with local rankers (Section 8.4) than in the inference model with global rankers (Section 8.5). Our hypothesis for what causes the global-ranking model to be less precision-biased is that in this configuration the ratio of errors on positive versus negative samples is more balanced. Thinking in the strategy that Perceptron follows, a local approach updates at every candidate with incorrect prediction sign, whereas a global approach only updates at candidates that should or should not be in the complete solution, after enforcing the domain constraints. In other words, the number of negative updates - which drives the precision bias - is reduced in the global approach, because some of the false positives generated by the ranking classifiers are eliminated by the domain constraints. Thus, because candidate scoring is trained to optimize accuracy, 


\begin{tabular}{|c|c|c|c|c|}
\hline WSJ & PProps & Prec. & Recall & $\mathrm{F}_{1}$ \\
\hline global feedback & $+3.10 \%$ & $-1.96 \%$ & $+1.99 \%$ & +0.27 \\
\hline $\max n$ & $+0.95 \%$ & $+0.67 \%$ & $+0.36 \%$ & +0.49 \\
\hline \multicolumn{5}{|l|}{ Brown } \\
\hline global feedback & $+2.62 \%$ & $-2.71 \%$ & $+2.25 \%$ & +0.38 \\
\hline max margin & $+1.00 \%$ & $+1.13 \%$ & $+0.55 \%$ & +0.78 \\
\hline
\end{tabular}

Table 13: Contribution of global feedback and max margin to the learning-based inference. The baseline is the "Pred-by-pred, local" model in Table 9.

fewer candidate arguments will be eliminated by the meta-learner with global rankers, which translates into a better balance between precision and recall.

Another important conclusion of our analysis of global versus local ranking for the learning-based inference is that a max-margin approach for the candidate scoring classifiers is more important than having global feedback for inference. In fact, considering that the only difference between the model with predicate-by-predicate inference with local feedback in Section 8.5 ("Pred-by-pred, local") versus the best model in Section 8.4 (+FS6) is that the latter uses SVM classifiers whereas the former uses Perceptron, we can compute the exact contribution of both max margin and global feedback ${ }^{15}$. For convenience, we summarize this analysis in Table 13. That table indicates that max margin yields a consistent improvement of both precision and recall, whereas the contribution of global feedback is more in reducing the difference between precision and recall by boosting recall and decreasing precision. The benefit of max-margin classifiers is even more evident in Table 12, which shows that the local-ranking model with max-margin classifiers generalizes better than the global-ranking model when the amount of training data is reduced significantly.

Even though in this paper we have analyzed several combination approaches with three independent implementations, the proposed models are in fact compatible with each other. Various combinations of the proposed strategies are immediately possible. For example, the constraint satisfaction model can be applied on the output probabilities of the candidate scoring component introduced in Section 7.2. Such a model eliminates the dependency on the output scores of the individual SRL models but retains all the advantages of the constraint satisfaction model, e.g., the formal framework to tune the balance between precision and recall. Another possible combination of the approaches introduced in this paper is to use max-margin classifiers in the learning-based inference with global feedback, e.g., by using a global training method for margin maximization such as SVMstruct (Tsochantaridis et al., 2004). This model would indeed have an increased training time ${ }^{16}$, but could leverage the advantages of both max-margin classifiers and inference with global feedback (summarized in Table 13). Finally, another attractive approach is stacking, i.e., $N$ levels of chained meta-learning. For example, we could cascade the learning-based inference model with global rankers, which boosts recall, with the learning-based inference with local rankers, which favors precision.

15. The contribution of global feedback is given by the model with joint inference and global feedback ("Full sentence, global") in Section 8.5.

16. This was the main reason why we chose Perceptron for the proposed online strategies. 


\section{Related Work}

The 4 best performing systems at the CoNLL-2005 shared task included a combination of different base subsystems to increase robustness and to gain coverage and independence from parse errors. Therefore, they are closely related to the work of this paper. The first four rows in Table 10 summarize their results under exactly the same experimental setting as the one used in this paper.

Koomen et al. (2005) used a 2 layer architecture close to ours. The pool of candidates is generated by: (a) running a full syntax SRL system on alternative input information (Collins parsing, and 5-best trees from Charniak's parser), and (b): taking all candidates that pass a filter from the set of different parse trees. The combination of candidates is performed in an elegant global inference procedure as constraint satisfaction, which, formulated as Integer Linear Programming, is solved efficiently. This is different from our work, where we "break" complete solutions from any number of SRL systems and we also investigate a meta-learning combination approach in addition to the ILP inference. Koomen et al.'s system was the best performing system at CoNLL-2005 (see Table 10).

Haghighi et al. (2005) implemented double re-ranking on top of several outputs from a base SRL model. The re-ranking is performed, first, on a set of $n$-best solutions obtained by the base system run on a single parse tree, and, then, on the set of best-candidates coming from the $n$-best parse trees. This was the second-best system at CoNLL-2005 (third row in Table 10). Compared to our decomposition and re-combination approach, the re-ranking setting has the advantage of allowing the definition of global features that apply to complete candidate solutions. According to a follow-up work by the same authors (Toutanova, Haghighi, \& Manning, 2005), these global features are the source of the major performance improvements of the re-ranking system. In contrast, we focus more on features that exploit the redundancy between the individual models, e.g., overlap between individual candidate arguments, and we add global information at frame level only from the complete solutions provided by individual models. The main drawback of re-ranking compared to our approach is that the different individual solutions can not be combined because re-ranking is forced to select a complete candidate solution. This implies that its overall performance strongly depends on the ability of the base model to generate the complete correct solution in the set of $n$-best candidates. This drawback is evident in the lower performance upper limit of the re-ranking approach (see Tables 3 and 11) and in the performance of the actual system - our best combination strategy achieves an $\mathrm{F}_{1}$ score over 2 points higher than Haghighi et al. in both WSJ and Brown ${ }^{17}$.

Finally, Pradhan, Hacioglu, Ward, Martin, and Jurafsky (2005b) followed a stacking approach by learning two individual systems based on full syntax, whose outputs are used to generate features to feed the training stage of a final chunk-by-chunk SRL system. Although the fine granularity of the chunking-based system allows to recover from parsing errors, we find this combination scheme quite ad-hoc because it forces to break argument candidates into chunks in the last stage.

17. Recently, Yih and Toutanova (2006) reported improved numbers for this system: $80.32 \mathrm{~F}_{1}$ for WSJ and 68.81 for Brown. However, these numbers are not directly comparable with the systems presented in this paper because they fixed a significant bug in the representation of quotes in the input data, a bug that is still present in our data. 
Outside of the CoNLL shared task evaluation, Roth and Yih (2005) reached the conclusion that the quality of the local argument classifiers is more important than the global feedback from the inference component. This is also one of the conclusions drawn by this paper. Our contribution is that we have shown that this hypothesis holds in a more complex framework: combination of several state-of-the-art individual models, whereas Roth and Yih experimented with a single individual model, only numbered arguments, and a slightly simplified problem representation: B-I-O over basic chunks. Additionally, our more detailed experiments allowed us to show clearly that the contribution of max margin is higher than that of global learning in several corpora and for several combinations of individual systems.

Punyakanok, Roth, and Yih (2005) showed that the performance of individual SRL models (particularly argument identification) is significantly improved when full parsing is used and argument boundaries are restricted to match syntactic constituents (similarly to our Model 3). We believe that the approach used by our Models 1 and 2, where candidate arguments do not have to match a single syntactic constituent, has increased robustness because it has a built-in mechanism to handle some syntax errors, when an argument constituent is incorrectly fragmented into multiple phrases. Our empirical results support this claim: Model 2 performs better than both Model 3 and the models proposed by Punyakanok et al. A second advantage of the strategy proposed in this paper is that the same model can be deployed using full syntax (Model 2) or partial syntax (Model 1).

Pradhan, Ward, Hacioglu, Martin, and Jurafsky (2005c) implement a SRL combination strategy at constituent level that, similarly to our approach, combines different syntactic views of the data based on full and partial syntactic analysis. However, unlike our approach, Pradhan et al.'s work uses only a simple greedy inference strategy based on the probabilities of the candidate arguments, whereas in this paper we introduce and analyze three different combination algorithms. Our analysis yielded a combination system that outperforms the current state of the art.

Previous work in the more general field of predicting structures in natural language texts has indicated that the combination of several individual models improves overall performance in the given task. Collins (2000) first proposed a learning layer based on ranking to improve the performance of a generative syntactic parser. In that approach, a reranker was trained to select the best solution from a pool of solutions produced by the generative parser. In doing so, the reranker dealt with complete parse trees, and represented them with rich features that exploited dependencies not considered in the generative method. On the other hand, it was computationally feasible to train the reranker, because the base method reduced the number of possible parse trees for a sentence from an exponential number (w.r.t. sentence length) to a few tens. More recently, global discriminative learning methods for predicting structures have been proposed (Lafferty, McCallum, \& Pereira, 2001; Collins, 2002, 2004; Taskar et al., 2003, 2004; Tsochantaridis et al., 2004). All of them train a single discriminative ranking function to detect structures in a sentence. A major property of these methods is that they model the problem discriminatively, so that arbitrary and rich representations of structures can be used. Furthermore, the training process in these methods is global, in that parameters are set to maximize measures not only related to local accuracies (i.e., on recognizing parts of a structure), but also related to the global accuracy (i.e., on recognizing complete structures). In this article, the use of global and rich representations is also a major motivation. 


\section{Conclusions}

This paper introduces and analyzes three combination strategies in the context of semantic role labeling: the first model implements an inference strategy with constraint satisfaction using integer linear programming, the second uses inference based on learning where the candidates are scored using discriminative classifiers using only local information, and the third and last inference model builds on the previous strategy by adding global feedback from the conflict resolution component to the ranking classifiers. The meta-learners used by the inference process are developed with a rich set of features that includes voting statistics -i.e., how many individual systems proposed a candidate argument- overlap with arguments from the same and other predicates in the sentence, structure and distance information coded using partial and full syntax, and probabilities from the individual SRL models (if available). To our knowledge, this is the first work that: (a) introduces a thorough inference model based on learning for semantic role labeling, and (b) performs a comparative analysis of several inference strategies in the context of SRL.

The results presented suggest that the strategy of decomposing individual solutions and performing a learning-based re-combination for constructing the final solution has advantages over other approaches, e.g., re-ranking a set of complete candidate solutions. Of course, this is a task-dependant conclusion. In the case of semantic role labeling, our approach is relatively simple since the re-combination of argument candidates has to fulfill only a few set of structural constraints to generate a consistent solution. If the target structure is more complex (e.g., a full parse tree) the re-combination step might be too complex from both the learning and search perspectives.

Our evaluation indicates that all proposed combination approaches are successful: they all provide significant improvements over the best individual model and several baseline combination algorithms in all setups. Out of the three combination strategies investigated, the best $F_{1}$ score is obtained by the learning-based inference using max-margin classifiers. While all the proposed approaches have their own advantages and drawbacks (see Section 8.7 for a detailed discussion of differences among the proposed inference models) several important features of a state-of-the-art SRL combination strategy emerge from this analysis: (i) individual models should be combined at the granularity of candidate arguments rather than at the granularity of complete solutions or frames; (ii) the best combination strategy uses an inference model based in learning; (iii) the learning-based inference benefits from max-margin classifiers and global feedback, and (iv) the inference at sentence level (i.e., considering all predicates at the same time) proves only slightly useful when the learning is performed also globally, using feedback from the complete solution after inference.

Last but not least, the results obtained with the best combination strategy developed in this work outperform the current state of the art. These results are empirical proof that a SRL system with good performance can be built by combining a small number (three in our experiments) of relatively simple SRL models.

\section{Acknowledgments}

We would like to thank the JAIR reviewers for their valuable comments.

This research has been partially supported by the European Commission (CHIL project, 
IP-506909; PASCAL Network, IST-2002-506778) and the Spanish Ministry of Education and Science (TRANGRAM, TIN2004-07925-C03-02). Mihai Surdeanu is a research fellow within the Ramón y Cajal program of the Spanish Ministry of Education and Science. We are also grateful to Dash Optimization for the free academic use of Xpress-MP.

\section{References}

Bishop, C. (1995). Neural Networks for Pattern Recognition. Oxford University Press.

Boas, H. C. (2002). Bilingual framenet dictionaries for machine translation. In Proceedings of LREC 2002.

Carreras, X., \& Màrquez, L. (2004). Introduction to the CoNLL-2004 shared task: Semantic role labeling. In Proceedings of CoNLL 2004.

Carreras, X., \& Màrquez, L. (2005). Introduction to the conll-2005 shared task: Semantic role labeling. In Proceedings of CoNLL-2005.

Carreras, X., Màrquez, L., \& Chrupała, G. (2004). Hierarchical recognition of propositional arguments with perceptrons. In Proceedings of CoNLL 2004 Shared Task.

Charniak, E. (2000). A maximum-entropy-inspired parser. In Proceedings of NAACL.

Collins, M. (1999). Head-Driven Statistical Models for Natural Language Parsing. PhD Dissertation, University of Pennsylvania.

Collins, M. (2000). Discriminative reranking for natural language parsing. In Proceedings of the 17th International Conference on Machine Learning, ICML-00, Stanford, CA USA.

Collins, M. (2002). Discriminative training methods for hidden markov models: Theory and experiments with perceptron algorithms. In Proceedings of the SIGDAT Conference on Empirical Methods in Natural Language Processing, EMNLP-02.

Collins, M. (2004). Parameter estimation for statistical parsing models: Theory and practice of distribution-free methods. In Bunt, H., Carroll, J., \& Satta, G. (Eds.), New Developments in Parsing Technology, chap. 2. Kluwer.

Collins, M., \& Duffy, N. (2002). New ranking algorithms for parsing and tagging: Kernels over discrete structures, and the voted perceptron. In Proceedings of the 40th Annual Meeting of the Association for Computational Linguistics, ACL'02.

Crammer, K., \& Singer, Y. (2003a). A family of additive online algorithms for category ranking. Journal of Machine Learning Research, 3, 1025-1058.

Crammer, K., \& Singer, Y. (2003b). Ultraconservative online algorithms for multiclass problems. Journal of Machine Learning Research, 3, 951-991.

Freund, Y., \& Schapire, R. E. (1999). Large margin classification using the perceptron algorithm. Machine Learning, 37(3), 277-296.

Gildea, D., \& Jurafsky, D. (2002). Automatic labeling of semantic roles. Computational Linguistics, 28(3). 
Gildea, D., \& Palmer, M. (2002). The necessity of syntactic parsing for predicate argument recognition. In Proceedings of the 40th Annual Conference of the Association for Computational Linguistics (ACL-02).

Hacioglu, K., Pradhan, S., Ward, W., Martin, J. H., \& Jurafsky, D. (2004). Semantic role labeling by tagging syntactic chunks. In Proceedings of the 8th Conference on Computational Natural Language Learning (CoNLL-2004).

Haghighi, A., Toutanova, K., \& Manning, C. (2005). A joint model for semantic role labeling. In Proceedings of CoNLL-2005 Shared Task.

Koomen, P., Punyakanok, V., Roth, D., \& Yih, W. (2005). Generalized inference with multiple semantic role labeling systems. In Proceedings of CoNLL-2005 Shared Task.

Lafferty, J., McCallum, A., \& Pereira, F. (2001). Conditonal random fields: Probabilistic models for segmenting and labeling sequence data. In Proceedings of the 18th International Conference on Machine Learning, ICML-01.

Marcus, M., Santorini, B., \& Marcinkiewicz, M. (1994). Building a large annotated corpus of English: The Penn Treebank. Computational Linguistics, 19(2).

Màrquez, L., Comas, P., Giménez, J., \& Català, N. (2005). Semantic role labeling as sequential tagging. In Proceedings of CoNLL-2005 Shared Task.

Melli, G., Wang, Y., Liu, Y., Kashani, M. M., Shi, Z., Gu, B., Sarkar, A., \& Popowich, F. (2005). Description of SQUASH, the SFU question answering summary handler for the DUC-2005 summarization task. In Proceedings of Document Understanding Workshop, HLT/EMNLP Annual Meeting.

Narayanan, S., \& Harabagiu, S. (2004). Question answering based on semantic structures. In International Conference on Computational Linguistics (COLING 2004).

Noreen, E. W. (1989). Computer-Intensive Methods for Testing Hypotheses. John Wiley \& Sons.

Palmer, M., Gildea, D., \& Kingsbury, P. (2005). The Proposition Bank: An annotated corpus of semantic roles. Computational Linguistics, 31(1).

Ponzetto, S. P., \& Strube, M. (2006a). Exploiting semantic role labeling, wordnet and wikipedia for coreference resolution. In Proceedings of the Human Language Technolgy Conference of the North American Chapter of the Association for Computational Linguistics.

Ponzetto, S. P., \& Strube, M. (2006b). Semantic role labeling for coreference resolution. In Companion Volume of the Proceedings of the 11th Meeting of the European Chapter of the Association for Computational Linguistics.

Pradhan, S., Hacioglu, K., Krugler, V., Ward, W., Martin, J. H., \& Jurafsky, D. (2005a). Support vector learning for semantic argument classification. Machine Learning, 60, 11-39.

Pradhan, S., Hacioglu, K., Ward, W., Martin, J. H., \& Jurafsky, D. (2005b). Semantic role chunking combining complementary syntactic views. In Proceedings of CoNLL-2005. 
Pradhan, S., Ward, W., Hacioglu, K., Martin, J. H., \& Jurafsky, D. (2005c). Semantic role labeling using different syntactic views. In Proceedings of the $43 \mathrm{rd}$ Annual Conference of the Association for Computational Linguistics.

Punyakanok, V., Roth, D., \& Yih, W. (2005). The necessity of syntactic parsing for semantic role labeling. In Proceedings of the International Joint Conference on Artificial Intelligence (IJCAI).

Punyakanok, V., Roth, D., Yih, W., \& Zimak, D. (2004). Semantic role labeling via integer linear programming inference. In Proceedings of the International Conference on Computational Linguistics (COLING'04).

Rosenblatt, F. (1958). The perceptron: A probabilistic model for information storage and organization in the brain. Psychological Review, 65, 386-407.

Roth, D., \& Yih, W. (2004). A linear programming formulation for global inference in natural language tasks. In Proceedings of the Annual Conference on Computational Natural Language Learning (CoNLL-2004), pp. 1-8, Boston, MA.

Roth, D., \& Yih, W. (2005). Integer linear programming inference for conditional random fields. In Proceedings of the International Conference on Machine Learning (ICML).

Schapire, R. E., \& Singer, Y. (1999). Improved boosting algorithms using confidence-rated predictions. Machine Learning, 37(3).

Surdeanu, M., Harabagiu, S., Williams, J., \& Aarseth, P. (2003). Using predicate-argument structures for information extraction. In Proceedings of the 41st Annual Meeting of the Association for Computational Linguistics (ACL 2003).

Taskar, B., Guestrin, C., \& Koller, D. (2003). Max-Margin Markov Networks. In Proceedings of the 17th Annual Conference on Neural Information Processing Systems, NIPS-03, Vancouver, Canada.

Taskar, B., Klein, D., Collins, M., Koller, D., \& Manning, C. (2004). Max-margin parsing. In Proceedings of the EMNLP-2004.

Toutanova, K., Haghighi, A., \& Manning, C. (2005). Joint learning improves semantic role labeling. In Proceedings of the 43rd Annual Meeting of the Association for Computational Linguistics (ACL'05), pp. 589-596, Ann Arbor, MI, USA. Association for Computational Linguistics.

Tsochantaridis, I., Hofmann, T., Joachims, T., \& Altun, Y. (2004). Support vector machine learning for interdependent and structured output spaces. In Proceedings of the 21st International Conference on Machine Learning, ICML-04.

Xue, N., \& Palmer, M. (2004). Calibrating features for semantic role labeling. In Proceedings of EMNLP-2004.

Yih, S. W., \& Toutanova, K. (2006). Automatic semantic role labeling. In Tutorial of the Human Language Technolgy Conference of the North American Chapter of the Association for Computational Linguistics.

Younger, D. H. (1967). Recognition and parsing of context-free languages in $n^{3}$ time. Information and Control, 10(2), 189-208. 